\title{
Radiation-induced dysfunction of energy metabolism in the heart results in the fibrosis of cardiac tissues
}

\author{
PENG XU ${ }^{1,2}$, YALI YI ${ }^{1,2}$, YIJING LUO ${ }^{3}$, ZHICHENG LIU ${ }^{3}$, YILIN XU ${ }^{1,2}$, \\ $\mathrm{JING} \mathrm{CAI}^{1}, \mathrm{ZHIMIN} \mathrm{ZENG}^{1,2}$ and ANWEN LIU ${ }^{1,2}$ \\ ${ }^{1}$ Department of Oncology, The Second Affiliated Hospital of Nanchang University; \\ ${ }^{2}$ Jiangxi Key Laboratory of Clinical Translational Cancer Research, Nanchang, Jiangxi 330006; \\ ${ }^{3}$ Department of Clinical Medicine, The First Clinical College of Nanchang University, Nanchang, Jiangxi 330031, P.R. China
}

Received February 27, 2021; Accepted June 29, 2021

DOI: $10.3892 / \mathrm{mmr} .2021 .12482$

\begin{abstract}
Thoracic radiotherapy increases the risk of radiation-induced heart damage (RIHD); however, the molecular mechanisms underlying these changes are not fully understood. The aim of the present study was to investigate the effects of radiation on the mouse heart using high-throughput proteomics. Male C57BL/6J mice were used to establish a model of RIHD by exposing the entire heart to $16 \mathrm{~Gy}$ high-energy $\mathrm{X}$-rays, and cardiac injuries were verified using a cardiac echocardiogram, as well as by measuring serum brain natriuretic peptide levels and conducting H\&E and Masson staining 5 months after irradiation. Proteomics experiments were performed using the heart apex of 5-month irradiated mice and control mice that underwent sham-irradiation. The most significantly differentially expressed proteins were enriched in 'cardiac fibrosis' and 'energy metabolism'. Next, the cardiac fibrosis and changes to energy metabolism were confirmed using immunohistochemistry staining and western blotting. Extracellular matrix proteins, such as collagen type $1 \alpha 1$ chain, collagen type III $\alpha 1$ chain, vimentin and CCCTC-binding factor, along with metabolism-related proteins, such as fatty acid synthase and solute carrier family 25 member 1 , exhibited upregulated expression following exposure to ionizing radiation. Additionally, the myocardial mitochondria inner membranes were injured, along with a decrease in ATP levels
\end{abstract}

Correspondence to: Dr Zhimin Zeng or Dr Anwen Liu, Department of Oncology, The Second Affiliated Hospital of Nanchang University, 1 Minde Street, Nanchang, Jiangxi 330006, P.R. China

E-mail: 2zm@163.com

E-mail: awliu666@163.com

Abbreviations: RIHD, radiation-induced heart damage; BNP, brain natriuretic peptide; IHC, immunohistochemistry; MHD, mean heart dose; DEPs, differentially expressed proteins; PPI, protein-protein interactions; ROS, reactive oxygen species; GO, Gene Ontology; FC, fold change; LVEF, left ventricle ejection fraction

Key words: RIHD, proteomics, energy metabolism, heart fibrosis, mitochondrial damage and the accumulation of lactic acid in the irradiated heart tissues. These results suggest that the high doses of ionizing radiation used lead to structural remodeling, functional injury and fibrotic alterations in the mouse heart. Radiation-induced mitochondrial damage and metabolic alterations of the cardiac tissue may thus be a pathogenic mechanism of RIHD.

\section{Introduction}

Thoracic radiotherapy is an important cancer treatment for patients diagnosed with malignant tumors, such as breast cancer, lung cancer, Hodgkin's lymphoma and esophageal cancer (1-4). Radiotherapy improves overall survival (OS) in patients with thoracic cancer, but it can result in some inevitable complications, particularly radiation-induced heart damage (RIHD), which has gradually become an increasing concern of oncologists and cardiologists $(5,6)$. The incidence of RIHD has increased due to the prolonged OS of patients with cancer, and RIHD usually occurs 5-10 years after radiation $(7,8)$. Radiation influences various aspects of the heart physiology, including the myocardium, pericardium, valves, coronary arteries and conduction system (9-11). Ionizing radiation exposure accelerates endothelial injury and facilitates the development of atherosclerotic plaques in coronary arteries clinically (10). The rates of major coronary events increase by $7.4 \%$ per gray as the mean heart dose (MHD) is increased, and these coronary events usually occur 5-20 years after radiotherapy amongst breast cancer survivors (12). It has been reported that the risk of coronary heart disease was increased 2.5-fold in a 20 Gy MHD group compared with patients with Hodgkin's lymphoma treated without radiation (2). Myocardial injury is another common complication of radiation. In a previous study, the prevalence of heart failure was nearly $24 \%$ at 20 years after receiving thoracic ionizing radiation (13). In preclinical studies, mice exposed to high doses of radiation often exhibited a decrease in left ventricular ejection fraction (LVEF) and an increase in expression of myocardial injury markers $(14,15)$. In our previous study, soluble suppression of tumorigenicity- 2 was identified as a cardiac biomarker that was shown to be increased amongst patients with cancer following thoracic radiotherapy, and it was positively associated with the radiation parameters $\mathrm{V}_{5}, \mathrm{~V}_{10}, \mathrm{~V}_{20}$ of heart and MHD (16). 
High-dose exposure ( $>5 \mathrm{~Gy}$ ) radiation, which is mostly observed in patients receiving radiation therapy, leads to cardiac injury (17). Low dose exposure $(<0.5 \mathrm{~Gy})$ radiation has also been investigated with cardiovascular effects, which is more frequently observed in Japanese atomic bomb survivors, Mayak workers or other nuclear workers (18). To further evaluate the heart damage of patients who received radiotherapy, the relevant literature was examined $(19,20)$, and consequently a high dose of 16 Gy was selected in the current study. In previous studies, RIHD progression was considered to be associated with energy deposition and the generation of reactive oxygen species (ROS), followed by molecular changes, damage to DNA, lipids and proteins, as well as the activation of early response transcription factors, cytokines, endothelial cell injury and signaling transduction pathways (21-25). This radiation stimulation to heart tissue of mice may result in increasing epicardial thickness and infiltrating inflammatory factors in the epicardium, as well as interstitial collagen deposition (20). To investigate RIHD in-depth, high-throughput proteomics analysis was used in the present study.

The latest advances in the high-throughput technologies of multiple 'combinatorial data' analyses may allow for a more direct and precise understanding of the molecular mechanisms underlying diseases $(26,27)$. Notably, proteomics are used to provide functional context in interpretation of genomic abnormalities, particularly in cancer biology $(28,29)$. Proteomics has gradually become an important technique in the fields of disease diagnosis, drug research and drug development, whilst also serving a predominant role in the study of the molecular basis of diseases and the understanding of various biological processes at the protein level $(30,31)$. Proteomics analysis of radiation injury may provide novel directions for the study of the mechanism underlying RIHD. Thus, in the present study, proteomics analysis was used to identify the changes that occurred in protein expression levels in cells or tissues.

The aim of the present study was to use a novel high-throughput proteomics technology to evaluate the effects of radiation on expression of proteins in the heart by establishing a RIHD mouse model to further understand the potential molecular mechanisms underlying the development of RIHD.

\section{Materials and methods}

Animal model of RIHD and local cardiac irradiation. In total, 18 C57BL/6 male mice (weight, 20-25 g; age, 8 weeks) were purchased from the Shanghai Institute of Biochemistry and Cell Biology. The mice were irradiated at the age of 8-9 weeks. All mice were maintained with controlled humidity (40-60\%) and temperature $\left(22-24^{\circ} \mathrm{C}\right)$ and a 12 -h light/dark cycle, with ad libitum access to food and water. The irradiated mice were sacrificed after 1,3 or 5 months, and the mice in the control group mice were sacrificed after 5 months. Mice were sacrificed by dislocating the cervical spine under intraperitoneal injection of $75 \mathrm{mg} / \mathrm{kg}$ pentobarbital sodium. Each group included 3-6 mice. Ethical approval for the animal experiments was obtained from the Institutional Review Board of The Second Affiliated Hospital of Nanchang University.

The whole heart was locally irradiated with a dose of 16 Gy using a precise small-animal radiation research platform (XStrahl Medical and Life Sciences) in the Zhejiang Key Radiation Laboratory. Mice in the control group were sham-irradiated and heart tissue was harvested after 5 months. All mice were anesthetized by intraperitoneal injection of $75 \mathrm{mg} / \mathrm{kg}$ pentobarbital sodium and placed in the supine position in the irradiation area of the small animal X-ray radiometer. The laser system was used to establish a 3D coordinate system. Irradiated mice underwent whole heart radiation. Control mice received sham irradiation (0 Gy). Cone-beam computed tomography, using $50 \mathrm{kV}$ and $0.8 \mathrm{~mA}$ photons filtered with aluminum $(1 \mathrm{~mm})$, was performed for each mouse to visualize the tomographic scanning of the thorax. Heart, lungs and the spinal cord were drawn by the same physicist during the tomographic scanning of the thorax, then the physicists designed and evaluated the radiotherapy plan, and limited the irradiated volume of the lung tissue and spinal cord in the mice as much as possible. A dose-volume histogram of the heart, lungs and spine was obtained (Fig. S1B). For arc radiation, a $0.15-\mathrm{mm}$ copper filter was fitted with a $10 \times 10 \mathrm{~mm}^{2}$ collimator $\left(-90^{\circ}\right.$ to $\left.90^{\circ}\right)$ at a source-skin distance of $342.5 \mathrm{~mm}$, energized at $220 \mathrm{kV}$ and $13.0 \mathrm{~mA}$ a single $\mathrm{X}$-ray beam with a dose rate of $2.32 \mathrm{~Gy} / \mathrm{min}$ (Fig. S1A, C-E).

Cardiac echocardiogram. Transthoracic echocardiography was performed using the Vevo 2100 ultrasound system (Visualsonics, Inc.) as described in our previous study (32). The mice were anesthetized by isoflurane (induction and maintenance doses of isoflurane were 3 and $1.5 \%$, respectively). Mice were placed in the supine position on an electrical heating pad at $37^{\circ} \mathrm{C}$. At the level of the largest left ventricle (LV), 2D guided M-mode echoes were obtained. The left ventricular posterior wall at the end of diastole was measured from the M-mode image in short axis. The LVEF and fractional shortening were calculated from the measured ventricle dimensions. Each group contained $\geq 3$ mice.

Measurement of serum brain natriuretic peptide (BNP) levels. Mice were anesthetized by intraperitoneal injection of $75 \mathrm{mg} / \mathrm{kg}$ pentobarbital sodium, and blood was collected from the vena orbital sinus of the 1-, 3- and 5-month irradiated mice group and the control group. Then, mice were sacrificed quickly via cervical dislocation. Blood samples ( $\sim 1 \mathrm{ml}$ per mouse) were collected in tubes with EDTA and the serum was separated via centrifugation at $4^{\circ} \mathrm{C}$ at $600 \mathrm{x} \mathrm{g}$ for $10 \mathrm{~min}$. BNP was determined using a high sensitivity ELISA kit [Presage BNP assay; USCNK, http://www.uscnk.cn/uscn /ELISA-Kit-for-Brain-Natriuretic-Peptide-(BNP)-49004.htm] according to the manufacturer's protocol. BNP levels were evaluated after determining the optical density of the samples at $450 \mathrm{~nm}$ (Thermo Fisher Scientific, Inc.). Each group contained $\geq 3$ mice.

$H \& E$, Masson staining and immunohistochemistry (IHC). Half of the apical part of mouse hearts in control $(n=3)$ and experiment $(n=3)$ group was quickly excised, immersed in $10 \%$ paraformaldehyde at room temperature for $24 \mathrm{~h}$ and embedded in paraffin. The heart tissue was cut into 5- $\mu \mathrm{m}$ thick sections, and the slides were stained with the H\&E staining kit (Beijing Solarbio Science \& Technology Co., Ltd.; cat. no. G1120). Masson staining of heart tissue was conducted 
using a Trichrome stain (Masson) kit (Beijing Solarbio Science \& Technology Co., Ltd.; cat. no. G1340) according to manufacturer's instructions.

IHC was performed as described previously (33). The $5-\mu \mathrm{M}$ sections were baked at $65^{\circ} \mathrm{C}$ for $30 \mathrm{~min}$, deparaffinized with xylene and rehydrated with a decreasing series of ethanol concentrations $(100,95,90,80$ and $70 \%)$. Then, antigen retrieval was performed using EDTA, after which tissues were treated with $3 \%$ hydrogen peroxide in methanol to quench endogenous peroxidase activity for $30 \mathrm{~min}$ at room temperature, and blocked with 5\% goat serum (Solarbio, Inc.) at room temperature for $1 \mathrm{~h}$. Primary antibodies against mouse anti-collagen type $1 \alpha 1$ chain (Col1a1; 1:500; ProteinTech Group, Inc.; cat. no. BA0325) or mouse anti-collagen type III $\alpha 1$ chain (Col3a1; 1:500; ProteinTech Group, Inc.; cat. no. M00788) were added, and samples were incubated at $4^{\circ} \mathrm{C}$ overnight, following which they were incubated with biotinylated secondary antibody (anti-rabbit; 1:5,000; OriGene Technologies, Inc.; cat. no. TA130017) for $1 \mathrm{~h}$ at room temperature and stained with DAB for $15 \mathrm{~min}$ at room temperature. After fully washing under tap water, sections were stained with $10 \%$ hematoxylin for $3 \mathrm{~min}$ at room temperature and sealed with neutral gum. Sections were examined using an Olympus CX33 light microscope (Olympus Corporation)

Proteomics analysis. Proteomics analysis was conducted and analyzed by Jingjie PTM Biolab Co., Ltd. The 5-month and control groups contained three mice each. The primary processes included protein extraction of heart apex with lysis buffer-8 $\mathrm{M}$ urea (Sigma-Aldrich; Merck KGaA), 1\% Protease Inhibitor (Calbiochem; Merck KGaA), $3 \mu$ M TSA (Sigma-Aldrich; Merck KGaA), 50 mM NAM (Sigma-Aldrich; Merck KGaA), 2 mM EDTA (Sigma-Aldrich; Merck KGaA), trypsin digestion and tandem mass tag (TMT) labeling, followed by high performance liquid chromatography (LC) fractionation and LC-mass spectrometry (MS)/MS analysis. MS/MS data were analyzed using the Maxquant search engine (https://www.maxquant. $\mathrm{org} /$ version 1.5.2.8), and the tandem mass spectra were analyzed using the SwissProt Mouse database (version 201808,16992 sequences, https://www.uniprot.org/), along with the reverse decoy database to calculate the false positive rate caused by random matching. Detailed descriptions can be found in the Data S1.

\section{Bioinformatics analysis}

Gene Ontology (GO) analysis. A GO annotation proteome was derived from the UniProt-GOA database (version Goa uniprot_gcrp.gaf.163; ebi.ac.uk/GOA/).

Subcellular localization analysis. Wolfpsort (version 0.2; genscript.com/psort/wolf_psort.html), a subcellular localization predication program, was used to predict the subcellular localization of the proteins.

Cluster of Orthologous Groups/Eukaryotic Orthologous Groups (COG/KOG) function classification. The KOG database (version 2003; https://ftp.ncbi.nih.gov/pub/COG/KOG/) was uses to classified DEPs for function annotations.

\section{Functional enrichment}

Enrichment of $G O$ analysis. GO annotations can be divided into three categories: Biological Process (BP), Cellular
Component (CC) and Molecular Function (MF), which categorize the biological functions of proteins based on different features. A two-tailed Fisher's exact test was used to assess the enrichment of the differentially expressed proteins (DEPs) against all identified proteins.

Enrichment of pathway analysis. The Kyoto Encyclopedia of Genes and Genomes (KEGG) database (version 2.5; https://www.kegg.jp/kegg/pathway) was used to study the enriched pathways using a two-tailed Fisher's exact test.

Enrichment of the protein domain analysis. InterPro (version 5.14-53.0; ebi.ac.uk/interpro/), a database providing functional analysis of protein sequences and predicting the presence of domains and important sites, was used and a two-tailed Fisher's exact test was used to assess the enrichment of the DEPs.

Protein-protein interaction (PPI) network mapping. All DEP database accession nos. or sequences were searched against the Search Tool for the Retrieval of Interacting Genes/Proteins (STRING) database version 11.0 (https://www. string-db.org/) to determine the PPI network. Only interactions between the proteins belonging to the searched dataset were selected, thereby excluding external candidates. STRING defines a metric termed 'confidence score' to describe the interaction confidence; all interactions that had a confidence score $>0.7$ (high confidence) were fetched. Interaction networks from STRING were visualized in Cytoscape 3.7.2 (34). Multi Contrast Delayed Enhancement (MCODE) version 1.5.1 was used to identify significant modules (MCODE score $\geq 10$ ) (35). Additionally, cytoHubba (version 1.6) was used to study essential nodes in the network using 11 methods [Density of maximum neighborhood component (DMNC) exhibits a satisfied comparative performance], and was completed to examine the hub genes (36).

Transmission electron microscopy. Parts of fresh apical heart portions of the mice after 5 months were quickly sectioned into $1-\mathrm{mm}^{3}$ cubes and fixed in paraformaldehyde (Beijing Solarbio Science \& Technology Co., Ltd.) for $2 \mathrm{~h}$ at $4^{\circ} \mathrm{C}$. Sections were fixed in $1 \%$ osmium tetroxide for $2 \mathrm{~h}$ at room temperature, followed by stepwise dehydration in graded acetone, after which, tissues were infiltrated, embedded (1:1 acetone/812 embedding agent for $3 \mathrm{~h}$ at $37^{\circ} \mathrm{C}, 1: 2$ acetone/812 embedding agent overnight at $37^{\circ} \mathrm{C}$ and 812 embedding agent for $6 \mathrm{~h}$ at $37^{\circ} \mathrm{C}$ ) and polymerized. The pieces were sectioned with an ultramicrotome into $1-2 \mu \mathrm{m}$ pieces, and then stained with a toluidine blue dye solution for $30 \mathrm{sec}$ at room temperature. The myocardial ultrastructure of the heart was observed on a HITACHI-7700 electron microscope (Hitachi, Ltd.). Each group contained three mice.

ATP and lactic acid assays. Samples of the apex of the heart were mixed with lytic fluid, homogenized with a homogenizer and then centrifuged at $4{ }^{\circ} \mathrm{C}$ at $12,000 \mathrm{x}$ g for $5 \mathrm{~min}$. ATP production was detected using an ATP Assay kit (Beyotime Institute of Biotechnology) according to the manufacturer's instructions. Relative light unit values were collected using with the Luminometer mode using a multimode Varioskan LUX microplate reader (Thermo Fisher Scientific, Inc.).

Lactic acid was detected using a lactic acid kit (Nanjing Jiancheng Bioengineering Institute) according to the 
manufacturer's protocol. The optical density value was measured using a multimode microplate reader at $530 \mathrm{~nm}$.

The protein concentration was measured using a BCA assay. Absolute ATP and lactate levels were calculated from the corresponding standard curve and normalized to the total protein concentration. Each group contained three mice.

Western blotting. Western blotting was performed using a standard protocol, as described previously (37). Samples of the apex of the heart from all mice were briefly homogenized using a homogenizer (PRO Scientific), and lysed in lysis buffer [50 mmol/1 Tris- $\mathrm{HCl}$ (pH 8.0), $150 \mathrm{mmol} / 1 \mathrm{NaCl}, 0.5 \% \mathrm{NP}-40$, $0.1 \%$ SDS and $5 \mathrm{mmol} / 1$ EDTA ( $\mathrm{pH} 8.0$ )] containing aprotinin phosphatase inhibitor (Beyotime Institute of Biotechnology) and protease inhibitor (Beyotime Institute of Biotechnology). Protein concentrations were quantified using a BCA Protein assay kit (Beyotime Institute of Biotechnology), and $10 \mu \mathrm{g}$ protein was loaded per a lane on an 8 or $12 \%$ SDS-gel. Proteins were resolved using SDS-PAGE and transferred to PVDF membranes (MilliporeSigma). The membranes were blocked using $5 \%$ fat-free milk for $1 \mathrm{~h}$ at room temperature and incubated with primary antibodies at $4^{\circ} \mathrm{C}$ overnight, followed by incubation with secondary antibodies (anti-rabbit; 1:5,000; Wuhan Boster Biological Technology, Ltd.) for $1 \mathrm{~h}$ at room temperature. Signals were visualized using chemiluminescence reagent (Bio-Rad Laboratories, Inc.) and semi-quantified using Image J 1.49 (National Institutes of Health). The primary antibodies used in the present study were: Anti-Collal polyclonal antibody (1:1,000; Wuhan Boster Biological Technology, Ltd.; cat. no. BA0325), anti-Col3a1 monoclonal antibody (1:1,000; Wuhan Boster Biological Technology, Ltd.; cat. no. M00788), anti-CCCTC-binding factor (CTGF) polyclonal antibody (1:1,000; Wuhan Boster Biological Technology, Ltd.; cat. no. PB0570), anti-vimentin (VIM) monoclonal antibody (1:1,000; Wuhan Boster Biological Technology, Ltd.; cat. no. BM0135), anti-fatty acid synthase (Fasn) monoclonal antibody (1:1,000; Wuhan Boster Biological Technology, Ltd.; cat. no. BM4865), anti-solute carrier family 25 member 1 (Slc25a1) polyclonal antibody (1:1,000; Wuhan Boster Biological Technology, Ltd. ; cat. no. A05995-2) and anti- $\alpha$-tubulin monoclonal antibody (1:1,000; Wuhan Boster Biological Technology, Ltd.; cat. no. M03989-2). The secondary antibodies used were anti-rabbit (1:10,000; cat. no. SA00001-2) and anti-mouse IgG (1:10,000; cat. no. SA00001-1), both of which were obtained from ProteinTech Group, Inc.

Statistical analysis. All data are presented as the mean \pm SD of $\geq 3$ repeats. Multiple comparisons difference involving $\geq 3$ groups were analyzed using one-way ANOVA with a Tukey's post hoc test and the differences between two groups were analyzed using unpaired Student's t-test. All analyses were performed using SPSS version 20 (IBM Corp.). $\mathrm{P}<0.05$ was considered to indicate a statistically significant difference.

\section{Results}

High-dose ionizing radiation causes cardiac structural remodeling and functional injury to the hearts of mice. To investigate the effects of high-energy X-rays on mouse heart tissue, an RIHD animal model was first established using local 16 Gy heart irradiation. Cardiac echocardiography, serum myocardial biomarkers, and H\&E and Masson staining were used to verify cardiac injury at the different time points. The results showed that LVEF and LV systolic posterior wall thickness were decreased, while LV systolic internal dimension was increased in the 5-month group compared with the 3-month group (Fig. 1A-G). Moreover, pericardial effusion was not observed in any of the groups (Fig. 1A-D). In addition, the levels of serum BNP, a cardiac biomarker, were significantly increased 5 months after exposure to ionizing radiation (Fig. $1 \mathrm{H})$.

H\&E staining of sections indicated that the cardiomyocytes had degenerated and the myofilament boundary was blurred after radiation, and these changes became more obvious as the time of follow-up increased (Fig. 2A). In addition, compared with the control mice, collagen fibers in the 5-month irradiated heart tissues were notably increased (Fig. 2B). Collectively, cardiomyocyte degeneration, fibrosis deposition, ventricular wall remodeling, BNP elevation and LV systolic dysfunction were evident in the 5-month irradiated mice. This indicated that myocardial damage and cardiac remodeling manifested in the mice in the 5 months after local heart radiation exposure.

\section{Proteomics analysis}

Protein quantification. In the aforementioned results, it was shown that mice manifested a RIHD phenotype 5 months after administration of $16 \mathrm{~Gy}$ ionizing radiation. Therefore, proteomics was performed using heart tissues from the control and 5-month irradiated mice (Fig. 3A). Principal component analysis and relative standard deviation were used to evaluate the quantitative repeatability of the proteins (Fig. 3B and C). A total of 269,637 secondary spectra were obtained by MS. After the secondary spectrum of the MS was analyzed using the protein theory database, the available number of spectra was 64,186 and the utilization rate of the spectrum was $23.8 \%$. A total of 29,161 peptides were identified by the spectral diagrams, amongst which, there were 28,194 unique peptides. From these peptides, 3,777 proteins were identified, and 3,274 were quantifiable (Fig. 3D). High-throughput proteomics analysis identified 234 proteins in total, and 219 proteins were found to be significantly increased [ $\log _{2}$ fold change $\left.(F C)>1.2\right]$, whereas only 15 proteins were considered downregulated $\left(\log _{2} \mathrm{FC}<1: 1.2\right)$ in the irradiated mouse hearts (Fig. 3E).

Subcellular localization and functional classification analysis. The 234 DEPs were searched in the WolfPsort database for prediction of their subcellular localization. The subcellular localization analysis found that the distributions of the DEPs were extracellular (46.58\%), cytoplasmic (18.8\%), nuclear $(14.1 \%)$, plasma membrane-bound (7.26\%), mitochondrial (6.41\%), endoplasmic reticulum (2.99\%) and cytoplasm, nucleus $(2.99 \%)$, with the remainder of proteins considered to be in other locations ( $0.85 \%$ ) (Fig. 4A).

According to the $\mathrm{GO}$ category results, the proteins related to BP were primarily 'cellular process', 'single-organism process', 'biological regulation', 'response to stimulus' and 'metabolic process', while CC was primarily composed of 'organelle', 'cell' and 'extracellular region' components. 'Binding' and 'catalytic activity' proteins were the primary MF (Fig. 4B). 


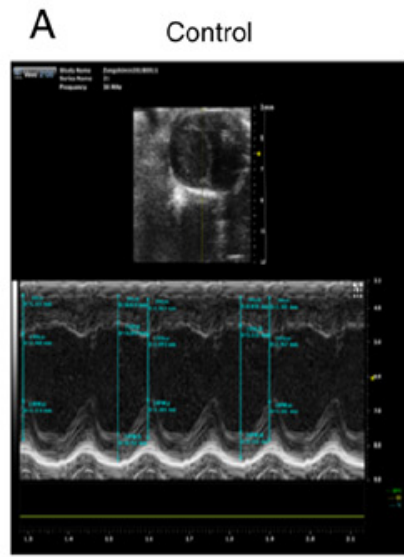

B

1 month

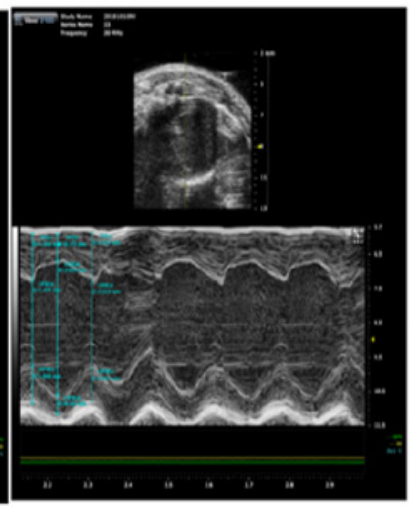

E
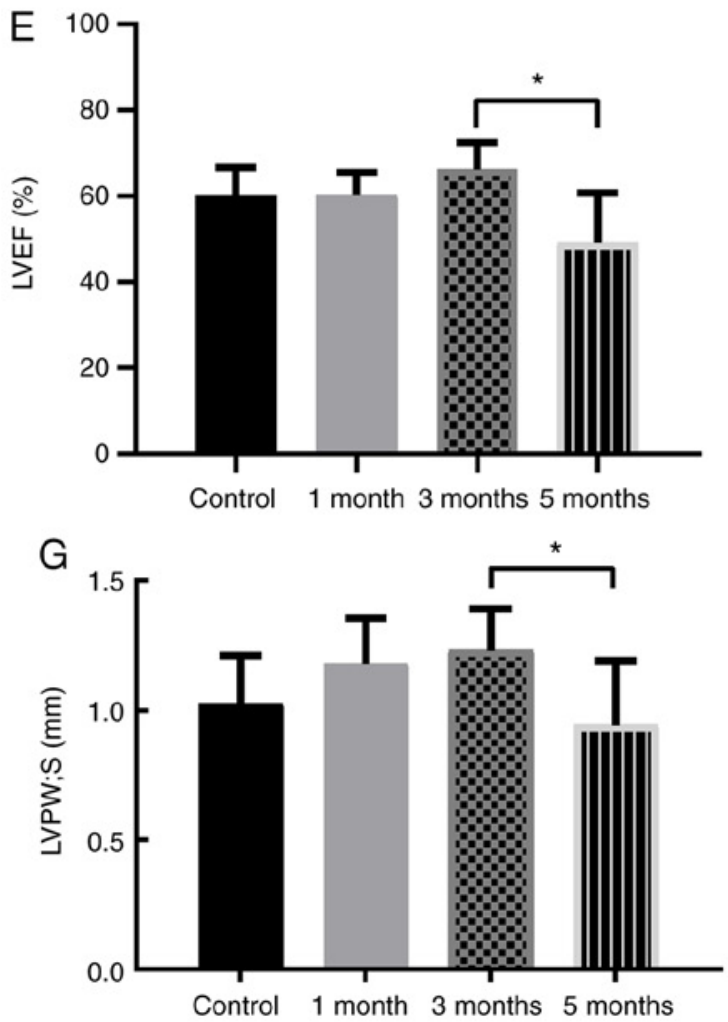

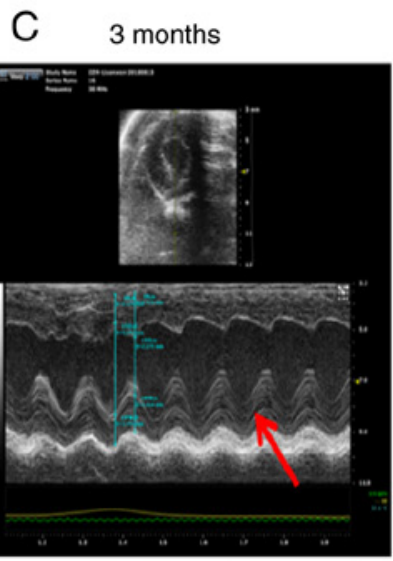

D 5 months

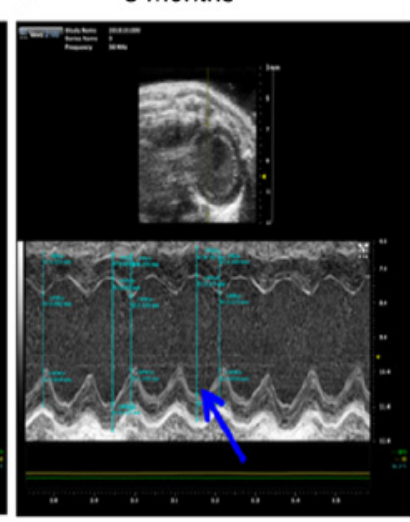

F

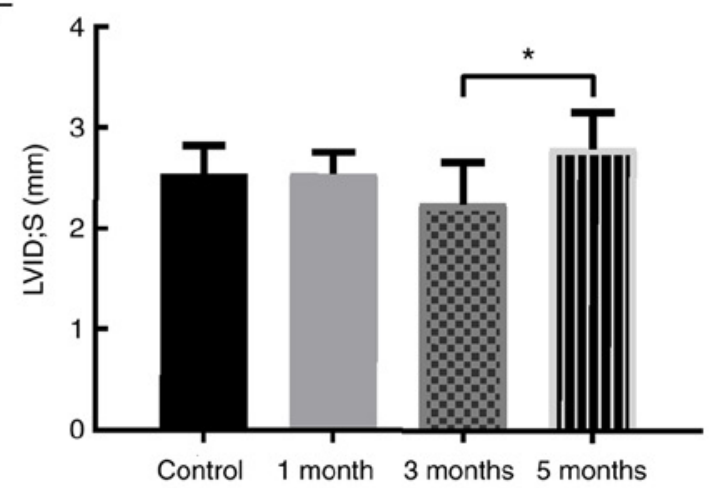

$\mathrm{H}$

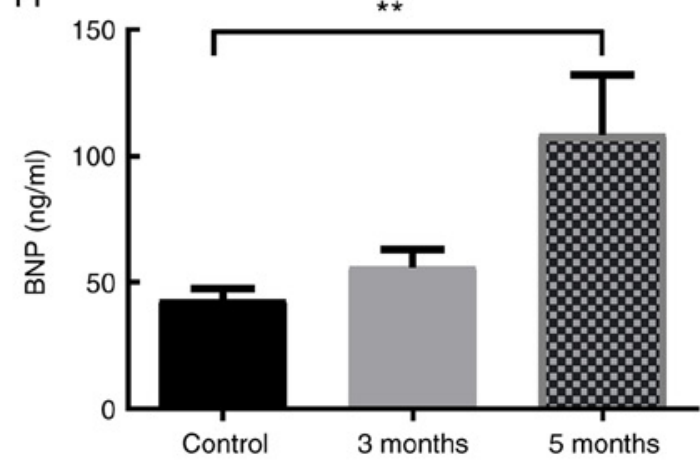

Figure 1. Local heart irradiation leads to RIHD after 5 months in mice. Representative images of mice echocardiographs in the (A) control mice, and mice (B) 1 , (C) 3 and (D) 5 months after local heart irradiation with a dose of 16 Gy. Red arrow, systolic thickness of the LV posterior wall 3 months after ionizing irradiation. Blue arrow, systolic thickness of the LV posterior wall 5 months after ionizing irradiation. (E) Cardiac parameter: LVEF (\%) in the control mice, and mice 1,3 and 5 months after local heart irradiation with a dose of $16 \mathrm{~Gy}$. (F) Cardiac parameter: LVID; $\mathrm{s}$ (mm) in the control mice, and mice 1, 3 and 5 months after local heart irradiation with a dose of $16 \mathrm{~Gy}$. (G) Cardiac parameter: LVPW; s (mm) in the control mice, and mice 1, 3 and 5 months after local heart irradiation with a dose of 16 Gy. (H) Serum BNP (ng/ml). ${ }^{*} \mathrm{P}<0.05,{ }^{* * *} \mathrm{P}<0.01 ; \mathrm{n}=3-6$ per group. LV, left ventricular; RIHD, radiation-induced heart damage; BNP, brain natriuretic peptide; LVID;s, Left ventricle internal dimension in systole; LVPW; s, LV posterior wall thickness in systole; LVEF, left ventricular ejection fraction.

The DEPs in the heart tissue induced by radiation were classified using COG/KOG (Fig. 4C). The results of the COG/KOG functional classification showed that the DEPs were primarily distributed in the following functions: (W) Extracellular structure, $(\mathrm{O})$ Posttranslational modification, protein turnover, chaperones. (T) Signal transduction mechanisms and (V) Defense mechanism. Moreover, there were also significant DEPs in substance metabolism after radiation, such as $(\mathrm{C})$ energy production and conversion, $(\mathrm{E})$ amino acid transport and metabolism, $(\mathrm{F})$ nucleotide transport and metabolism, $(\mathrm{G})$ carbohydrate transport and metabolism and (I) lipid transport and metabolism (Fig. 4C). Thus, metabolism-related proteins were significantly altered according to their subcellular localization and functional classification analysis.

Functional enrichment analysis. To determine the significant enrichment trend of the DEPs amongst the functional types, the enrichment of the DEPs in the irradiated group compared with the control group were analyzed through three aspects: GO term enrichment, KEGG pathway enrichment and protein domain enrichment.

The BP analysis results of the GO secondary classification showed that 'collagen fibril organization', 'protein activation cascade' and 'plasma membrane invagination' were amongst the most apparent differences. Abundant proteins were mainly 
A
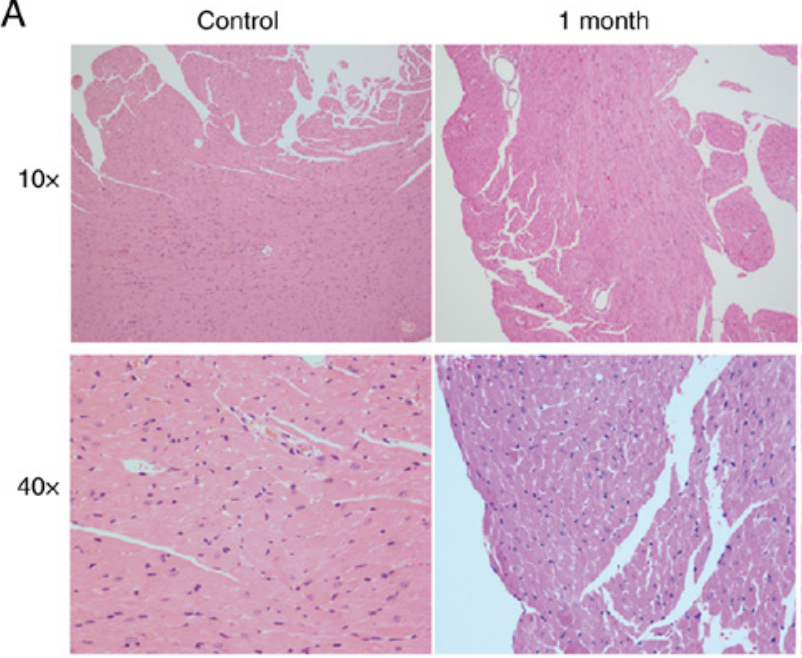

B
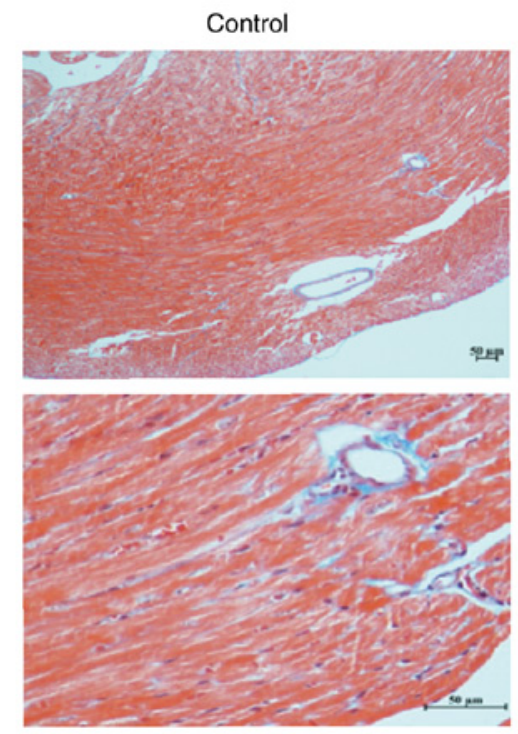

3 months
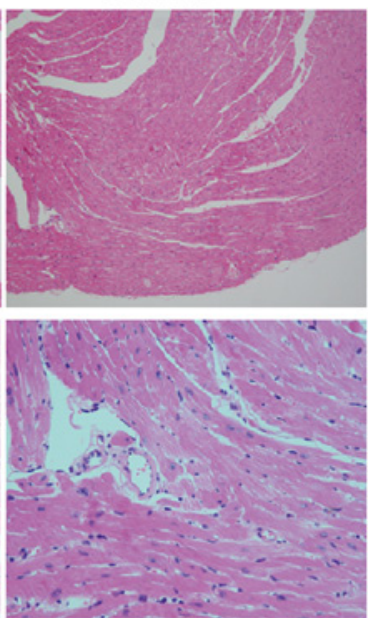

5 months
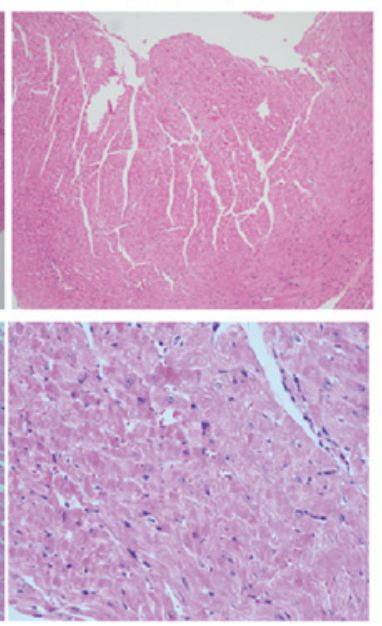

3 months
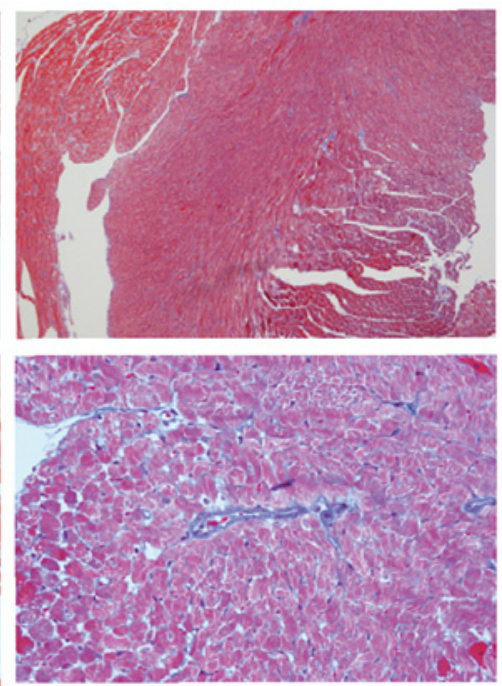

5 months
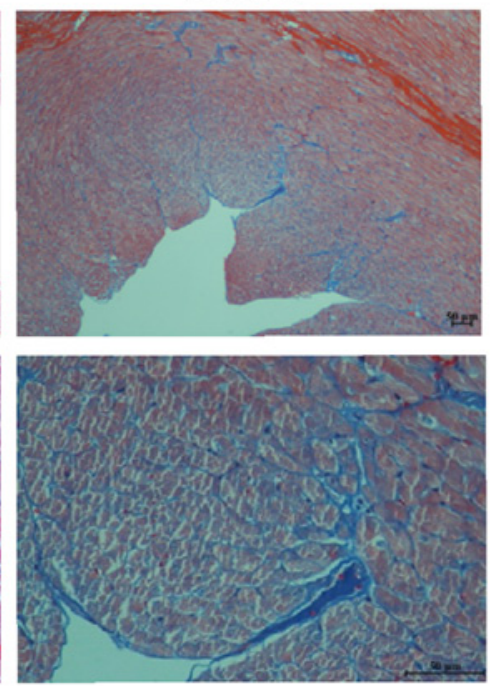

Figure 2. H\&E and Masson staining of locally irradiated and control mouse heart tissues. (A) H\&E staining of heart tissues from the control mice, and 1,3 and 5-month groups. Upper panel, light microscope at magnification, 10x10; lower panel, light microscope at magnification, 10x40. (B) Masson's staining of heart tissues from the control mice, and 1, 3 and 5-month groups. Upper panel, light microscope at magnification, 10x10; lower panel, light microscope at magnification, $10 x 40$.

enriched in 'negative regulation of protein metabolic process' and 'regulation of proteolysis' (Fig. 5A). CC enrichment analysis demonstrated that the proteins related to 'fibrillar collagen trimer', 'banded collagen fibril', 'extracellular matrix components', 'extracellular vesicles', 'extracellular region' or the parts of extracellular structures were increased significantly after irradiation (Fig. 5B). MFs, such as 'platelet-derived growth factor binding', 'immunoglobulin receptor binding', 'serine-type endopeptidase inhibitor activity', 'extracellular matrix structural constituent', 'peptidase regulator activity' and 'enzyme inhibitor activity' were also significantly increased after irradiation (Fig. 6A).

KEGG enrichment analysis found that the radiation-induced altered proteins were primarily enriched in 'Staphylococcus aureus infection', 'complement and collagen cascade', 'protein digestion and absorption', 'extracellular matrix (ECM) interactions' and 'folding and adhesion related signaling pathways' (Fig. 6B). The domains of these differentially expressed proteins were primarily enriched in 'immunoglobulin subtypes', 'immunoglobulin folding', 'EGF-like domains' and 'immunoglobulin-like domains', amongst others (Fig. S2). From the three functional enrichment analyses, fibrosis was considered to be increased following irradiation.

PPI network analysis. In order to identify the significant clusters (confidence score $>0.7$ ), 74 proteins were filtered into the DEP PPI network complex using STRING, and the resultant PPI network contained 74 nodes and 870 edges (Fig. 7A). MCODE was used to identify the most significant module that was comprised of two clusters (Fig. 7B and C) that consisted of upregulated DEPs (MCODE score $>10$ ). The top 10 hub genes selected using the DMNC method (score $\geq 5,000$ ) and node degree (score $\geq 10$ ) in the cytoHubba plug-in included transthyretin, GC vitamin D binding protein, $\alpha-1$-microglobulin/bikunin precursor, $\alpha-1$-antitrypsin $1-4$, inter- $\alpha$-trpsin inhibitor heavy chain 1 , histidine rich glycoprotein, serine protease inhibitor A3M, hemopexin (Hpx), serpin family F member 2 and complement factor I (Fig. 7D). The 
A

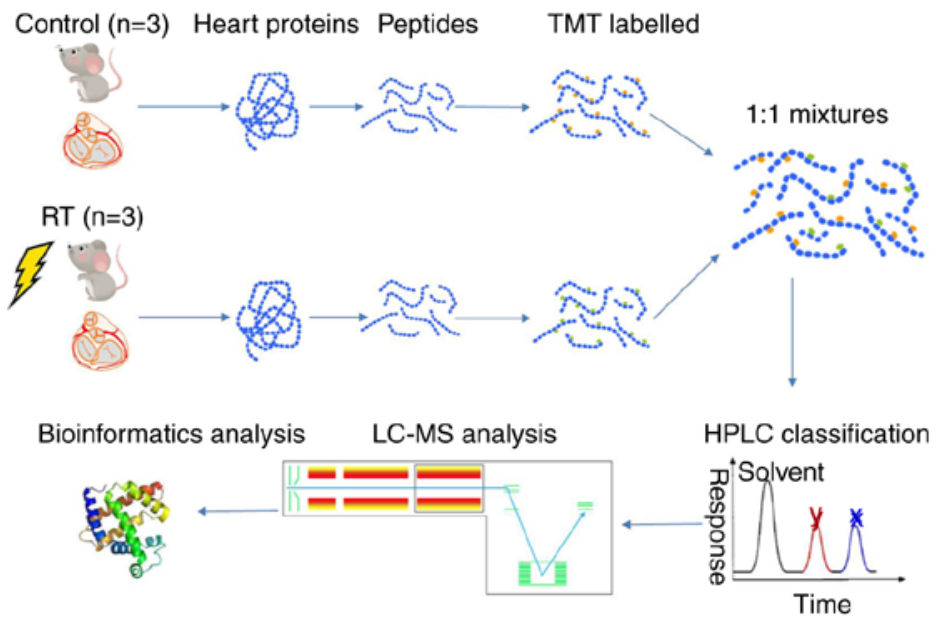

B

Groups $\rightarrow$ Con $\rightarrow$ Exp

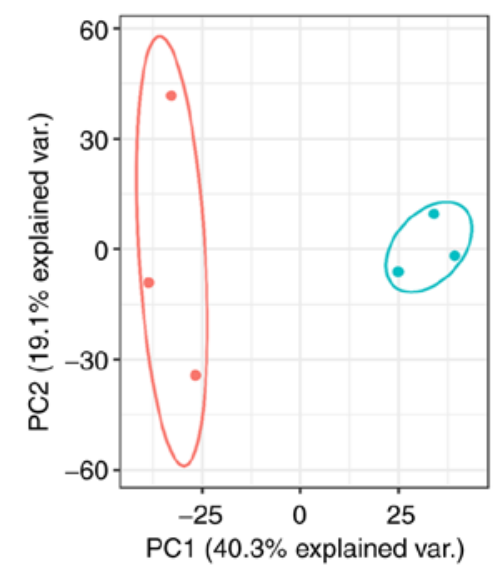

C

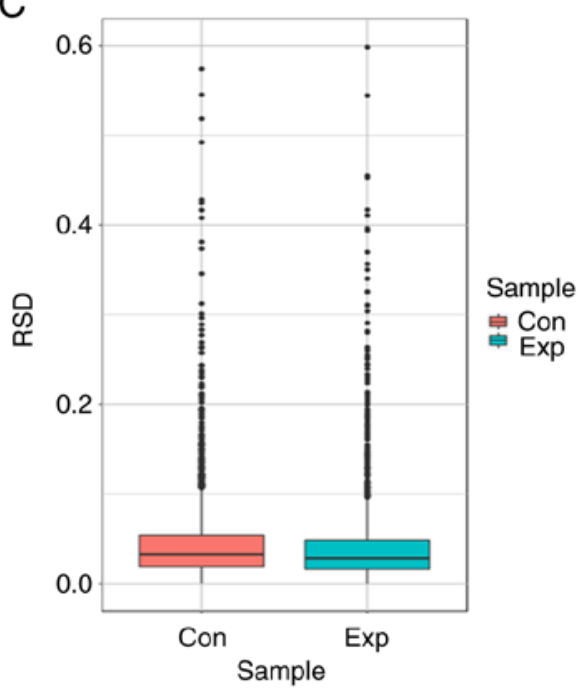

D

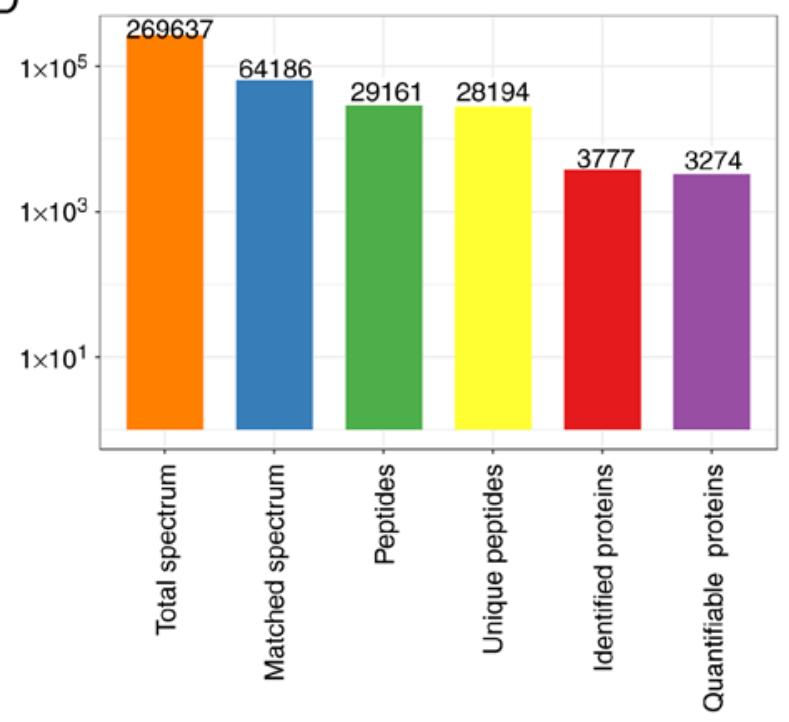

E

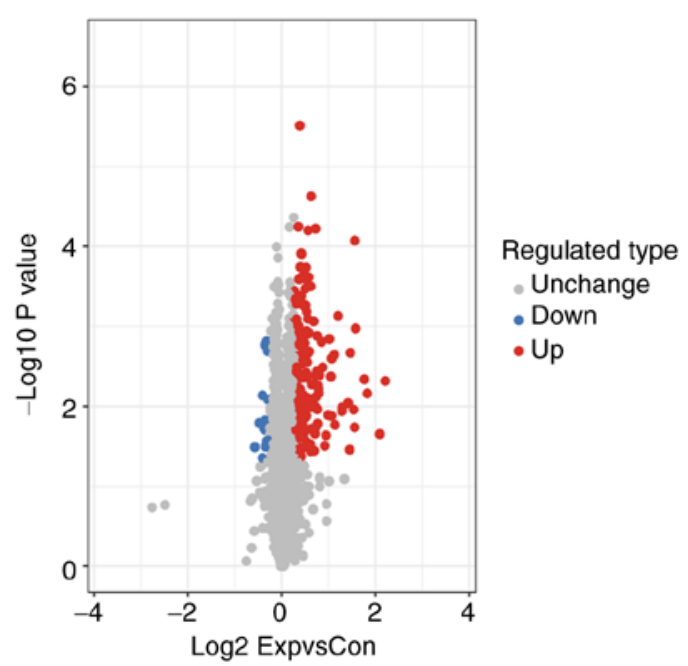

Figure 3. Proteomics analysis and protein quantification. (A) LC-MS/MS analysis procedure. (B) 2D scatter plots of the principal component analysis distribution of all samples using the quantified proteins. (C) Box plots of the RSD distribution of the repeated samples using the quantified proteins. (D) Statistical analysis of the MS results. (E) Volcano plot of the DEPs. n=3 per group. RT, radiotherapy; DEP, differentially expressed protein; LC-MS, liquid chromatography-mass spectrometry; TMT, tandem mass tag; HLPC, high performance liquid chromatography; RSD, relative standard deviation; con, control; Exp, experiment.

results of MCODE and cytoHubba analysis indicated that core DEPs were associated with abnormal lipid metabolism, negative regulation of fibrinolysis and ECM (Tables SI-SVI).
Irradiation induces ECM and mitochondrial metabolism damage. According to the proteomics analysis results, it was found that irradiation led to the upregulation of ECM proteins, 
A

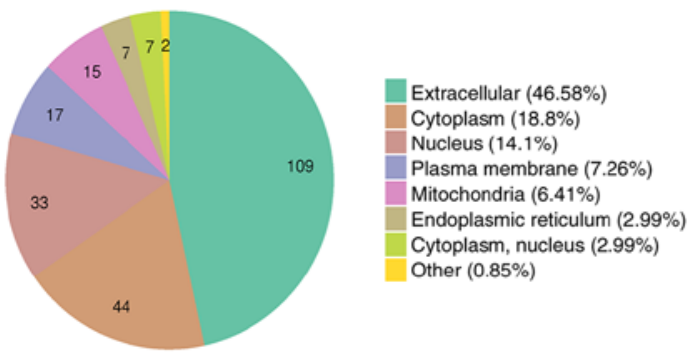

C

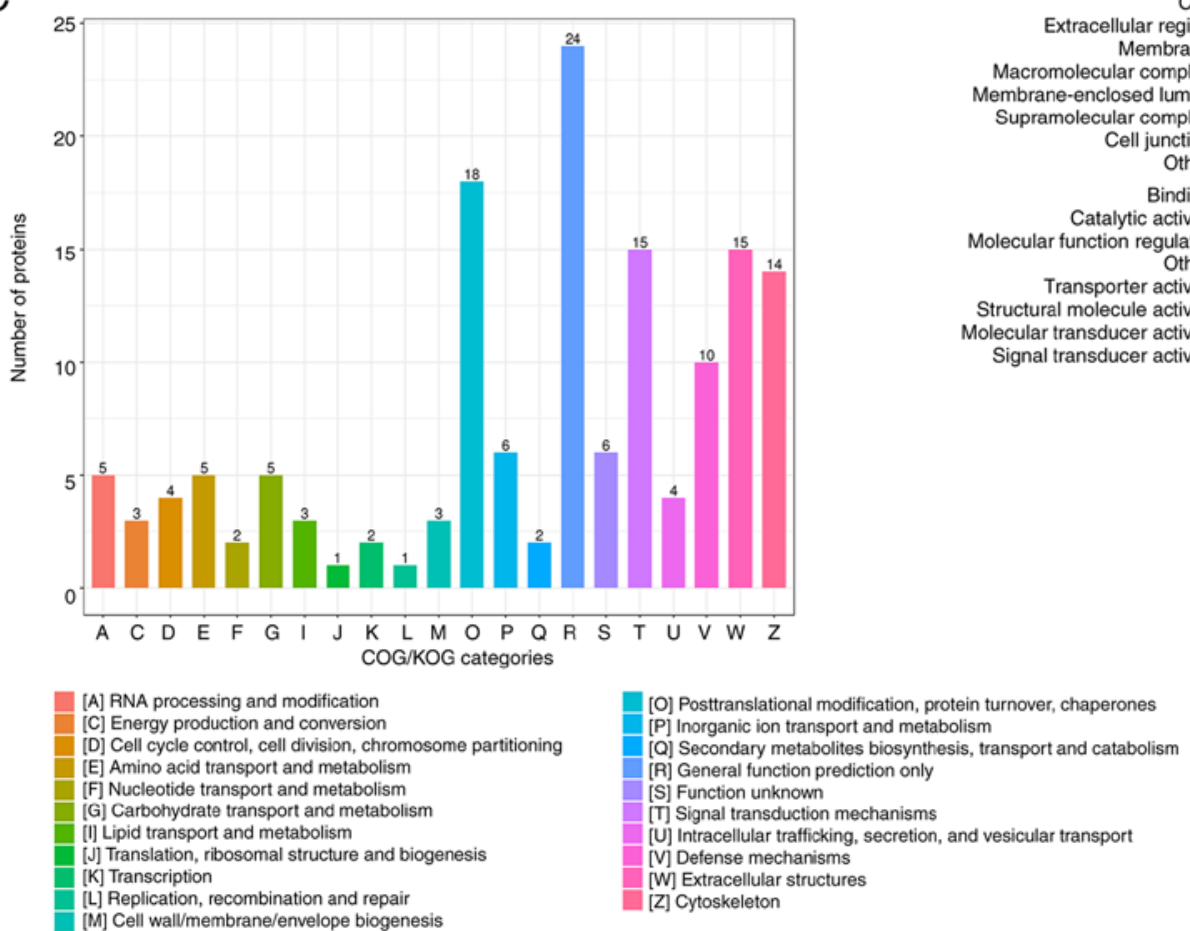

B

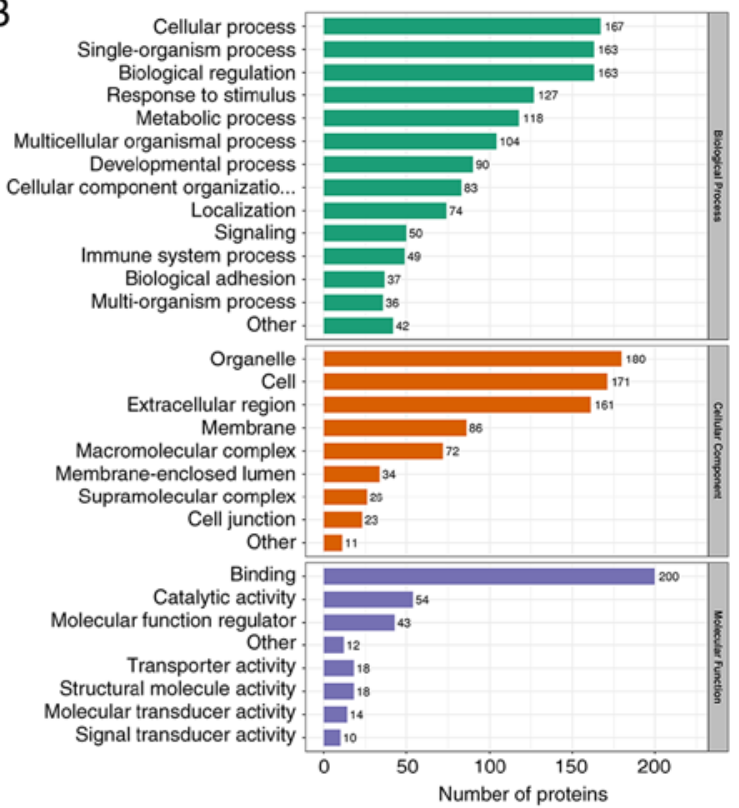

Figure 4. Subcellular localization and functional classification. (A) Subcellular localization chart of the DEPs. (B) Statistical distribution chart of the DEPs under each GO category. (C) COG/KOG functional classification chart of the DEPs. Three biological repetitions per group. DEP, differentially expressed protein; GO, Gene Ontology; COG/KOG, Cluster of Orthologous Groups/Eukaryotic Orthologous Groups.

such as Col14a1, perisostin (Postn), galectin 3 (Lgals3), Hpx, Tgf $\beta$ i, Col2a1, Col5a2, Col3a1, Col1a2, Col1a1, SPARC like 1 (Sparcl1), Col5a1, vitronectin (Vtn), Col6a1 and laminin subunit $\alpha 5$ (Lama5) (Table I). Colla1 and Col3a1 in the irradiated heart were shown to be upregulated, as determine by IHC and western blotting experiments (Fig. 8A-C). Additionally, other fibrosis-associated proteins (Vim and CTGF) were also upregulated (Fig. 8B and C). The results of the proteomics analysis indicated that metabolism-related proteins associated with carbohydrate transport and metabolism, energy production and conversion, amino acid transport and metabolism, nucleotide transport metabolism and lipid transport metabolism were increased (Table II). Metabolism-related proteins, such as Fasn and Slc25a1 were confirmed to be overexpressed by western blotting (Fig. 8D and E).

Subsequently, the electron micrograph results of the 5-month irradiated heart mitochondria were found to have myofilaments with fuzzy boundaries, swelling and cavitation of the mitochondria were observed, and fewer mitochondria were present compared with control group (Fig. 8F). The 3-month irradiated heart mitochondria electron micrograph results are shown in Fig. S3. It was also found that ATP production was decreased following irradiation, from $133.2 \mu \mathrm{mol} / \mathrm{gprot}$ in the control to $103.0 \mu \mathrm{mol} / \mathrm{gprot}$ in the irradiated mice (Fig. 8G). However, lactate production in the irradiated heart tissue was notably increased from $110.7 \mu \mathrm{mol} / 1$ in the control to $269.4 \mu \mathrm{mol} / 1$ in the irradiated mice (Fig. $8 \mathrm{H}$ ).

\section{Discussion}

The aim of the present study was to elucidate the potential mechanisms involved in RIHD in mice using a novel high-throughput proteomics technology. Using this technology, 29,161 peptides were identified, including 28,194 specific peptides, and 3,274 quantitative proteins amongst the 3,777 identified proteins. Of these, 219 proteins were significantly upregulated and 15 proteins were downregulated. By contrast, Azimzadeh et al (38) investigated the proteomics of 16-week-old irradiated mouse heart (16 Gy) and found that there were 662 myocardial proteins identified and 371 quantified proteins. Moreover, Subramanian et al (39) studied the proteomics of the mouse hearts at 40 weeks after 16 Gy radiation, and identified 1,038 proteins, of which 940 proteins were quantified. In the present study, 3,777 proteins were screened 
A
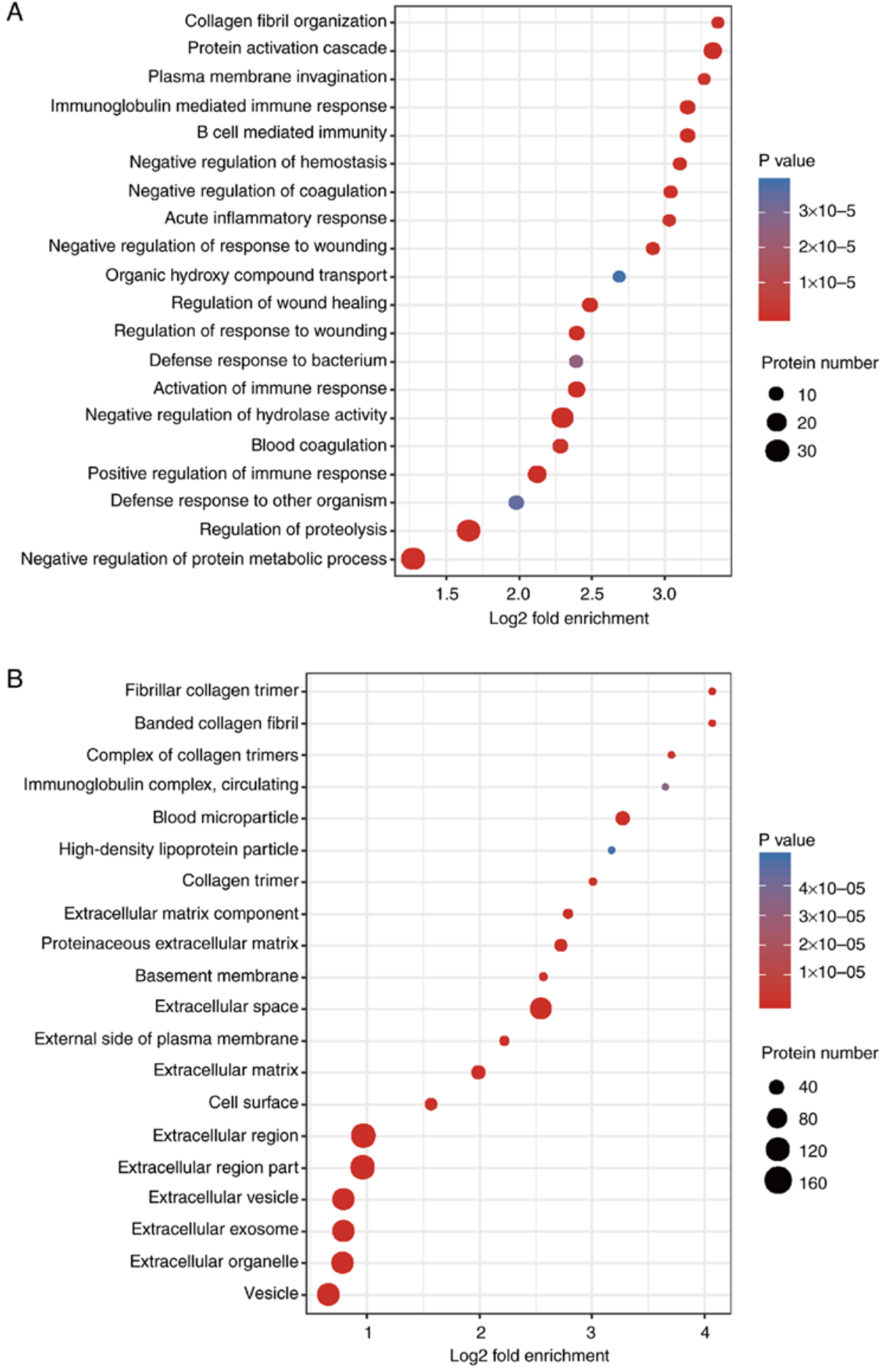

Figure 5. Functional enrichment analysis. GO enrichment bubble plot of the (A) Biological Process and (B) Cellular Component of the DEPs. $n=3$ per group. GO, Gene Ontology; DEP, differentially expressed protein.

and 3,274 proteins were quantified using TMT labeling, which may be the highest flux and labeling rate in the historical data of RIHD, and this increased quantity of data may further show potential mechanisms associated with RIHD.

The majority of these altered proteins were extracellular proteins (46.58\%). Fibrosis is the abnormal deposition of ECM, which can lead to organ dysfunction, morbidity and necrosis (40-42). According to COG/KOG functional classification, 15 upregulated proteins associated with the ECM or fibrosis were increased; these included Col14a1, Postn, Lgals3, Hpx, Tgfbi, Col2a1, Col5a2, Col3a1, Col1a2, Col1a1, Sparcl1, Col5a1, Vtn, Col6a1 and Lama5. According to GO enrichment analysis, the results of $\mathrm{BP}$ and $\mathrm{CC}$ classifications indicated that there were significant changes in fibrotic proteins. Additionally, protein domain enrichment, KEGG pathway enrichment and PPI analysis showed that radiated injury was associated with the ECM and fibrillar collagen. Intriguingly, the most significantly correlated enriched pathway based on KEGG 

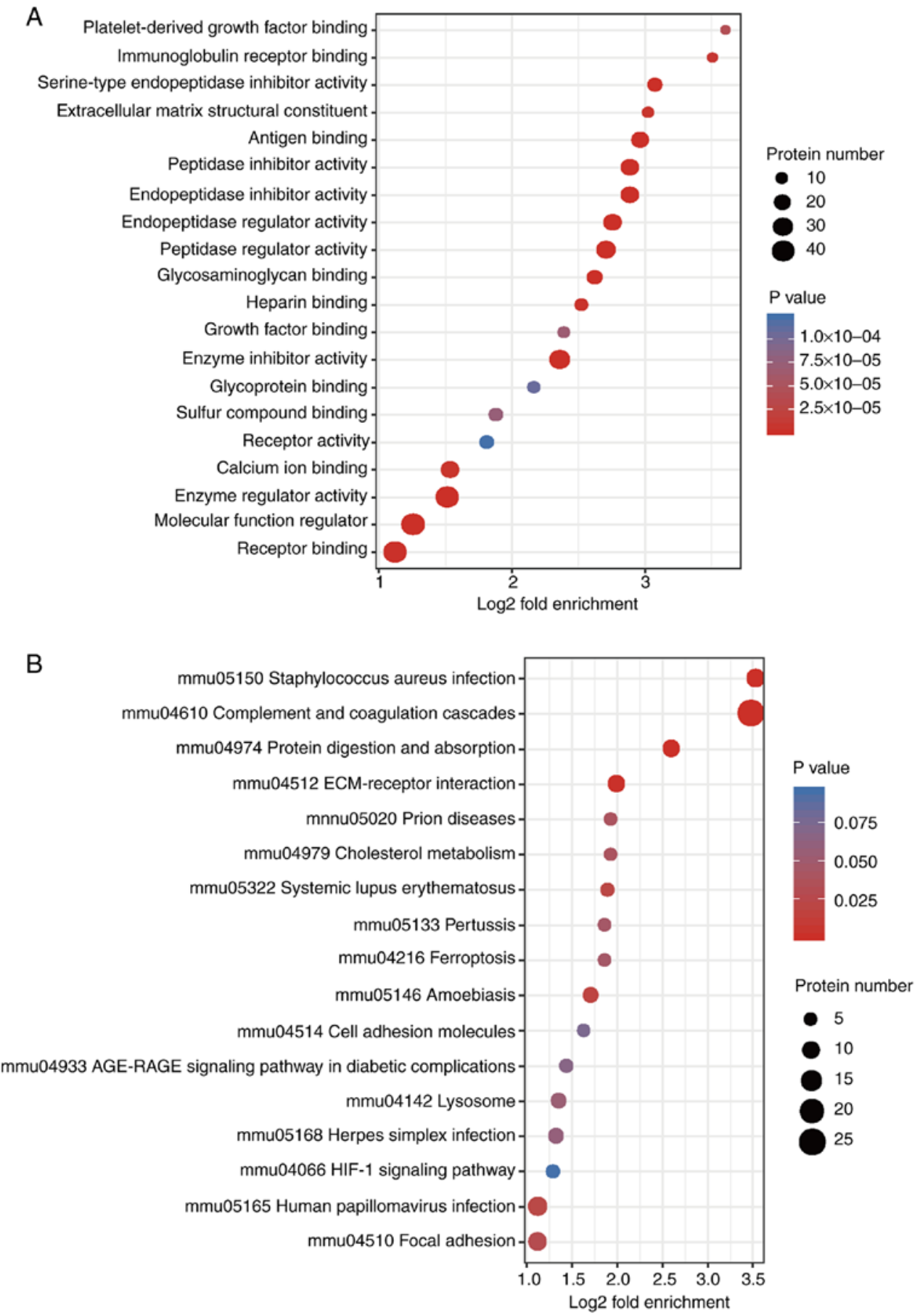

Figure 6. Functional enrichment analysis. (A) GO enrichment bubble plot of the Molecular Functions of the DEPs. (B) KEGG pathway enrichment bubble plot of the DEPs. $n=3$ per group. GO, Gene Ontology; KEGG, Kyoto Encyclopedia of Gene and Genomes; DEP, differentially expressed protein; ECM, extracellular matrix.

pathway analysis was 'Staphylococcus aureus' infection. Stoddard et al (43) used molecular techniques to investigate the microbial flora in chronic sinusitis caused by head-and neck radiotherapy, and found that Staphylococcus aureus was the most common microorganism present following radiation. Moreover, the microbiota induces alterations of metabolism and inflammatory responses following radiotherapy (44). The change in the flora of microbiota may lead to alterations in metabolism and the inflammatory response of irradiated heart tissues, although this requires further analysis.

It has been reported that the mechanism via which RIHD occurs may be associated with endothelial cell injury, inflammatory reactions and $\operatorname{ROS}(9,21,25)$. Irradiation leads to endothelial cell injury via pro-inflammatory cytokines and damage of DNA or proteins mediated by ROS. Endothelial dysfunction influences vascular intimal collagen deposition, which can cause vessel wall thickening and luminal stenosis (45). A series of pathological effects, such as ischemia and hypoxia of the irradiated heart, may aggravate the inflammatory reaction (25). With the continuous release of inflammatory cytokines, such as TNF, IL-1, IL-6 and IL-8, cardiac parenchyma cell necrosis and excessive deposition of ECM is observed in irradiated heart tissue (9). Clinically, the rate of chronic infections and fibrosis are increased following 

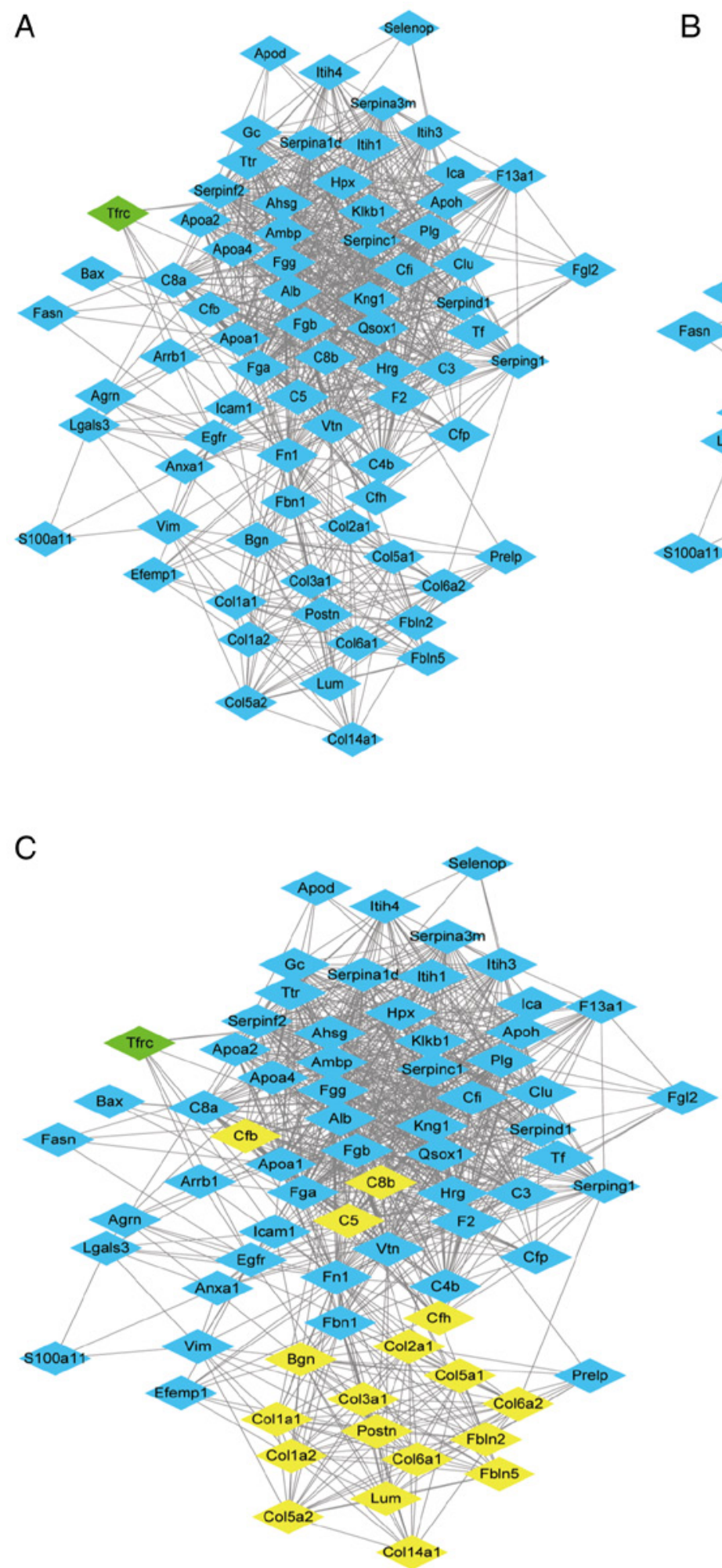

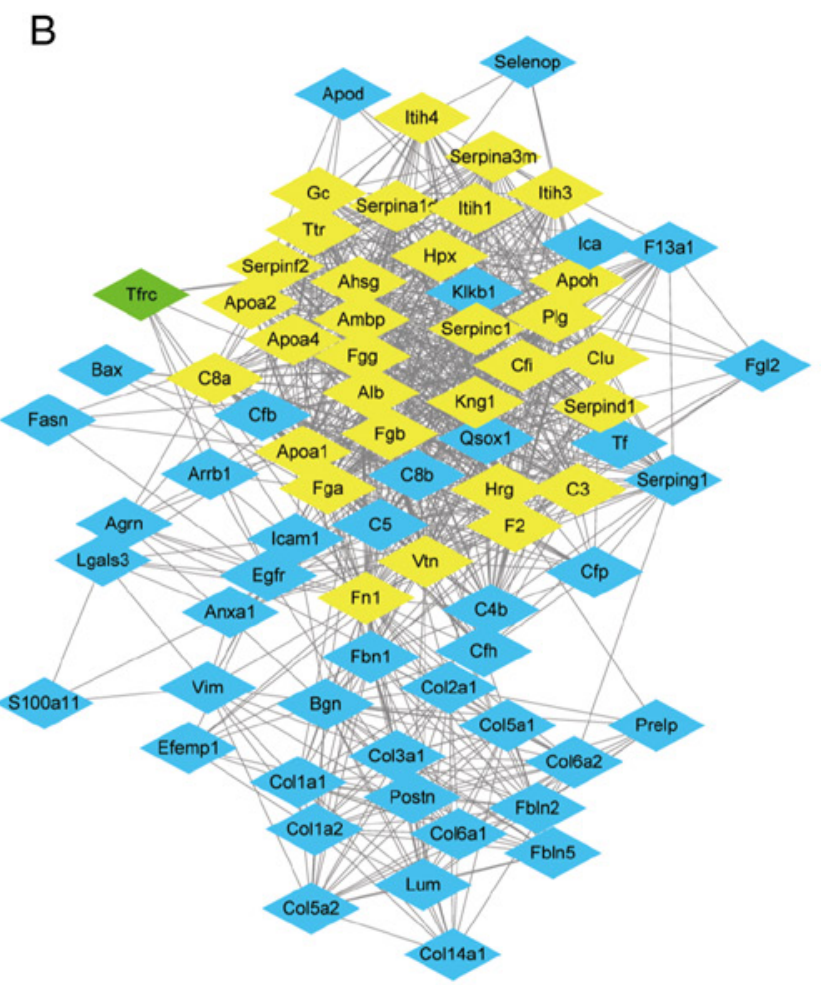

D

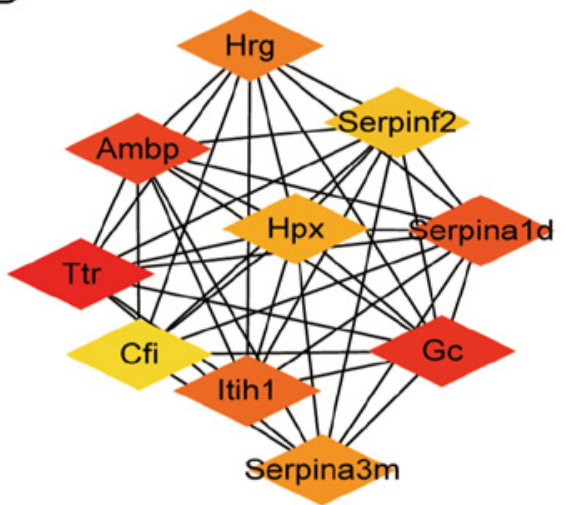

Figure 7. PPI network. (A) PPI network of the DEPs. Blue nodes represent upregulated genes, and green nodes represent downregulated genes. (B and C) MCODE was used to extract the most highly interconnected functional clusters from the network, which are indicated in yellow. (D) cytoHubba was used to extract top 10 hub proteins from the PPI network. Node colors reflect the degree of connectivity. Red nodes represent a higher degree of connectivity, and yellow nodes represent a lower degree of connectivity. PPI, protein-protein Interaction; DEP, differentially expressed protein; MCODE, MultiContrast Delayed Enhancement.

thoracic radiation therapy and Staphylococcus aureus infections (46). These clinical results are consistent with the proteomic data in the present study, in that high-energy radiation can indeed cause inflammation, ECM and fibrosis.

In recent years, metabolism-associated disorders have become increasingly recognized as important pathogenic processes of several diseases, including neurological disorders, type 2 diabetes mellitus, cancer and organ fibrosis, amongst others $(47,48)$. In the present study, COG/KOG functional classification of high-throughput proteomics indicated that proteins associated with various metabolic processes were increased. Mitochondria are best known as the sites of production of 
A
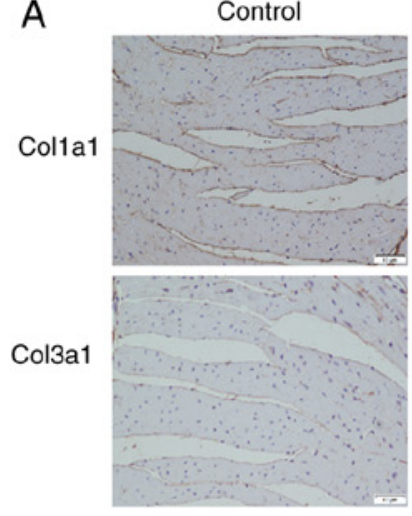

C

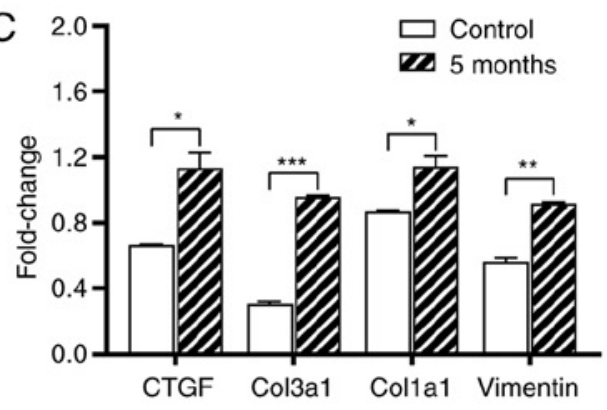

F

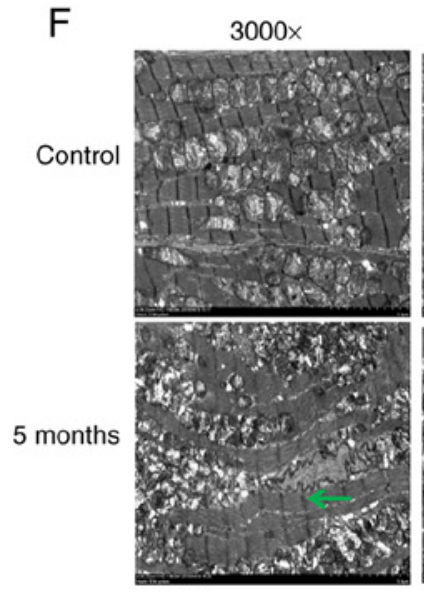

3 months
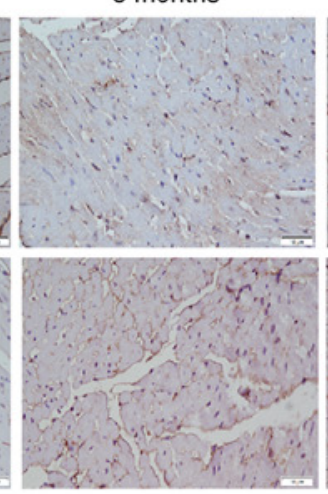
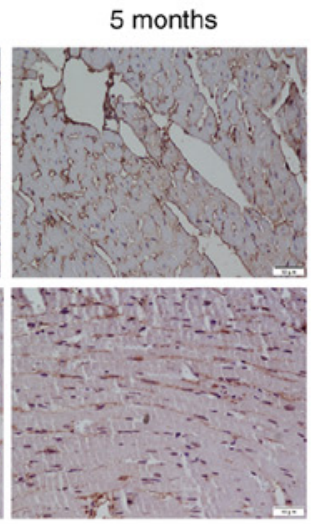

B

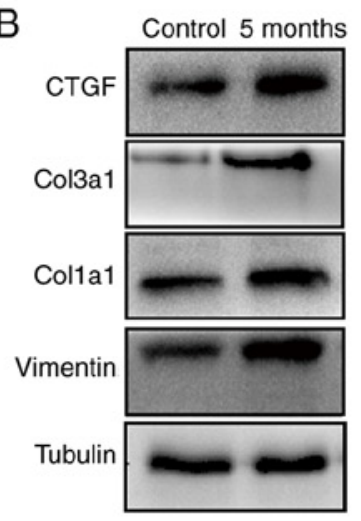

D

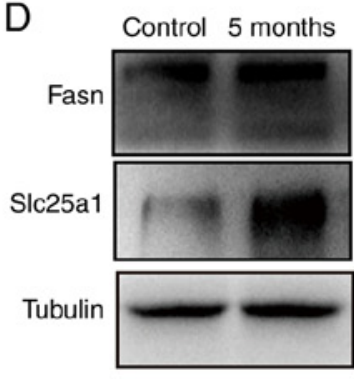

E

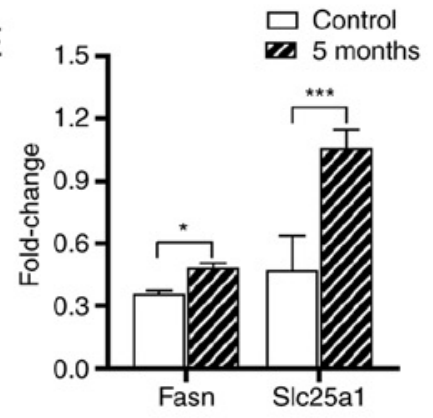

G
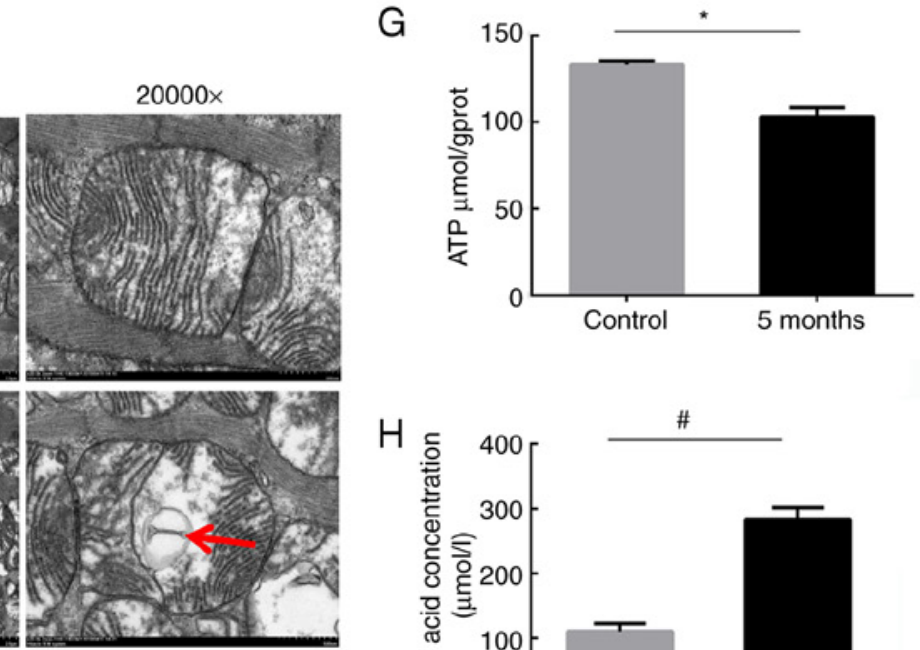

$6000 \times$

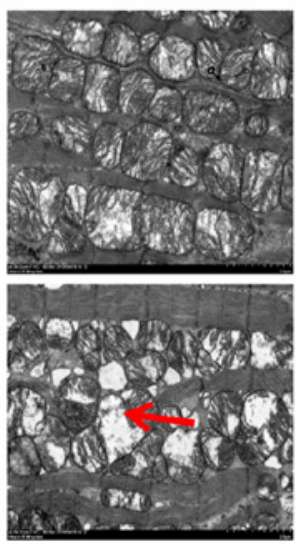

-

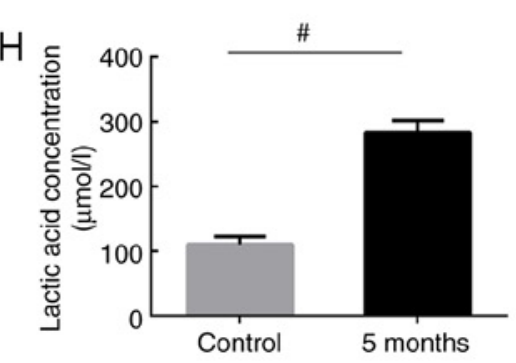

Figure 8. Irradiation induces alterations to the extracellular matrix and mitochondrial metabolism. (A) Immunohistochemistry staining analysis of Colla1 and Col3a1 in the heart tissues. Magnification, x400. (B) Western blotting and (C) semi-quantitative analysis of Col1a1, Col3a1, Vimentin and CTGF. (D) Western blotting and (E) semi-quantitative analysis of metabolism related genes Fasn and Slc25al. Relative protein levels of Colla1, Col3a1, Vimentin, CTGF, Fasn and Slc25al were normalized to Tubulin. (F) Electron micrographs of the cardiac mitochondria from the mice in sham-irradiated and 5-month groups after 16 Gy radiation. Left magnification, x3,000; Middle magnification, x6,000; Right magnification, x20,000. Green arrows show the myofilaments with fuzzy boundaries. Red arrows show swollen mitochondria with cavitation. Measurement of $(\mathrm{G})$ ATP levels and $(\mathrm{H})$ lactic acid concentration in the sham-irradiated and 5-month mice heart tissues. ${ }^{*} \mathrm{P}<0.05,{ }^{* * *} \mathrm{P}<0.01,{ }^{* * *} \mathrm{P}<0.001 ;{ }^{*} \mathrm{P}<0.05 ; \mathrm{n}=3$ per group. Colla1, collagen type $1 \alpha 1$ chain; Col3a1, collagen type III $\alpha 1$ chain; CTGF, CCCTC-binding factor; Fasn, fatty acid synthase; Slc25a1, solute carrier family 25 member 1.

respiratory ATP, and are essential for metabolic process $(49,50)$. Electron micrographs of the mitochondria were obtained in the present study, and ATP levels and lactate production of the irradiated heart tissue were measured. High-dose ionizing radiation resulted in increased structural alterations of cardiac mitochondria. Consistent with the histological results, changes in the inner membrane of the mitochondria led to alterations in expression of metabolism-related enzymes.
Several ECM- and metabolism-related proteins have been reported to be upregulated in irradiated heart tissue. Previous studies have highlighted the metabolic processes promoting factors, including increasing glycine, ATP synthesis and mitochondria respiration, involved in the pathogenesis of fibrosis (51-53). Glucose metabolism can provide energy for anabolic processes and collagen production. It is well established that not only glucose metabolism, but also protein and lipid metabolism are 
Table I. Upregulation of extracellular matrix proteins in mouse hearts 5 months after exposure to ionizing radiation.

\begin{tabular}{llccc}
\hline Category & Protein name & Ratio & Regulated type & P-value \\
\hline Extracellular structures & Col14a1 & 1.832 & Up & 0.0032566 \\
& Postn & 1.643 & Up & 0.0184358 \\
Lgals3 & 1.637 & Up & 0.0100236 \\
Hpx & 1.531 & Up & 0.0092418 \\
Tgfbi & 1.468 & Up & 0.0008027 \\
Col2a1 & 1.419 & Up & 0.0005821 \\
Col5a2 & 1.406 & Up & 0.0023836 \\
Col3a1 & 1.342 & Up & 0.0002423 \\
Col1a2 & 1.334 & Up & 0.0001224 \\
Col1a1 & 1.331 & Up & 0.0004185 \\
Sparcl1 & 1.314 & Up & 0.043479 \\
Col5a1 & 1.299 & Up & 0.0041625 \\
Vtn & 1.287 & Up & 0.0076994 \\
Col6a1 & 1.24 & Up & 0.0013383 \\
Lama5 & 1.219 & Up & 0.0004591 \\
& & &
\end{tabular}

Table II. Upregulation of metabolism-related proteins in the mouse heart 5 months after exposure to ionizing radiation.

\begin{tabular}{lllcc}
\hline Category & Protein name & Ratio & Regulated type & P-value \\
\hline [C] Energy production and conversion & Slc25a1 & 1.228 & Up & 0.0136395 \\
& Aldh1a1 & 1.268 & Up & 0.0105642 \\
& Aldh1b1 & 1.257 & Up & 0.013897 \\
[G] Carbohydrate transport and metabolism & Hexb & 1.245 & Up & 0.0017016 \\
& Manba & 1.311 & Up & 0.038884 \\
& Slc2a1 & 1.309 & Up & 0.0061217 \\
& Tkt & 1.217 & Up & 0.0160025 \\
[E] Amino acid transport and metabolism & Cfi & 1.318 & Up & 0.0001813 \\
& Abat & 1.335 & Up & 0.0140643 \\
& Cfb & 1.429 & Up & 0.0005422 \\
& Klkb1 & 1.605 & Up & 0.0008644 \\
[F] Nucleotide transport and metabolism & Lta4h & 1.204 & Up & 0.0122628 \\
& Dpysl3 & 1.247 & Up & 0.0009192 \\
[I] Lipid transport and metabolism & Entpd1 & 1.232 & Up & 0.022776 \\
& Fasn & 1.617 & Up & 0.0052028 \\
& Lta4h & 1.204 & Up & 0.0122628 \\
& Ttr & 1.444 & Up & 0.0011445 \\
\hline
\end{tabular}

closely correlated with mitochondrial function (54-56). Indeed, mitochondria are considered to be the major suppliers of cellular energy in the form of ATP, which is produced by oxidative phosphorylation, and is the primary means of energy production under baseline conditions (57,58). Vincent et al (59) investigated keloid scars with excessive amounts of collagen production, and concluded that keloid-associated fibroblasts consume unusually large amounts of glucose and produce more lactate to fulfill their ATP needs, which means the secretion of collagen relies on ATP produced by glycolysis. However, in normal heart tissue, fatty acids are the main source of energy metabolism, accounting for $40-80 \%(60,61)$.
Metabolic changes are regarded as important pathogenic processes of fibrosis in various organs, and metabolic targeted therapy may become an important strategy to reduce fibrosis $(41,49,62)$. Disturbance of myocardial energy metabolism is an important cause of myocardial heart disease, and mitochondria are the primary organelles involved in energy metabolism $(50,63,64)$. Similarly, mitochondrial dysfunction may also be an important cause of radiation-induced heart disease. It has been shown that the respiratory capacity of myocardial mitochondria among C57BL/6 mice was significantly decreased 40 weeks after local 2 Gy radiation, but not in the 0.2 Gy group (12). Sridharan et al (65) used Sprague 
Dawley rats to establish a radiation and tyrosine kinase inhibitors-related heart injury model and showed enhanced effects on mitochondrial morphology and mitochondrial permeability transition pore opening. Another study confirmed that late application of a palladium lipoate complex improved myocardial mitochondrial function by reducing the presence of a cellular inflammatory microenvironment, although it had no effect on repairing the damaged mitochondrial structures (66). In 2019, Dai et al (67) also reported that radiation caused damage to heart function, and this was mediated by ROS produced by the mitochondria. The simple ganoderma lucidum spore oil system these authors developed targeted the myocardial mitochondria and slowed down the production of ROS, thus exhibiting a preventative and therapeutic effect on RIHD. Additionally, the differences in expression levels of mitochondrial genes caused by genetic variants may lead to differences in sensitivity to ionizing radiation (68). Collectively, the aforementioned studies revealed that mitochondrial damage was an important target in RIHD, and drugs or drug-loaded materials for the repair of mitochondrial damage can reduce radiation-induced heart injury.

Radiation induces oxidative changes and persistent inflammation last days and months after the initial exposure, possibly due to the continuous generation of ROS and nitrogen species (69). Defects in mitochondrial DNA, protein import, proteins and metabolic enzymes resulting from exposure to ionizing radiation may lead to late health effects, including degenerative diseases and metabolic disorders (23). In the present study, via electron microscopic analysis of mitochondria it was indicated that myocardial mitochondria damage persisted after high dose radiation. Therefore, the mitochondrial damage caused by ionizing radiation may be a significant cause of the pathogenic mechanisms underlying RIHD.

The present study has some limitations. The heart tissue samples for proteomics were obtained from the heart apex, including the left ventricle and right ventricle. Thus, it is hard to distinguish the LV alternations alone. Additionally, the study was performed in male mice only, and differences in sex should be considered.

In the present study, the DEPs between irradiated and control mice heart tissue, as well as the alterations enriched in fibrosis and energy metabolism, were identified. Ionizing radiation indeed led to cardiac fibrosis and metabolic disorders, and these were validated by subsequent experiments. No direct mechanism was identified in the present study; however, further investigations regarding the mechanisms between ATP alterations and radiation induced cardiac fibrosis are ongoing.

In conclusion, the results of the present study demonstrated that ionizing radiation caused structural remodeling, functional injury and fibrotic alterations in the heart. Radiation-induced mitochondrial damage and metabolic alterations in cardiac tissue may underlie the pathogenic mechanisms of RIHD.

\section{Acknowledgements}

We thank physicist Dr Peng Zhang (Zhejiang Key Laboratory of Radiation Oncology) for supporting our work of establishing the animal model.

\section{Funding}

This study was supported by the National Natural Science Foundation of China (grant nos. 81760566 and 82060577), Science and Technology Innovation Platform of Jiangxi Province (grant no. 20171BCD40022), Development Project of Jiangxi province (grant no. 20171ACB20034), Project in The Second Affiliated Hospital of Nanchang University (grant no. Y578\#) and Postgraduates Special Funds Innovation of Jiangxi Province (grant no. YC2020-B048).

\section{Availability of data and materials}

The datasets used and/or analyzed during the current study are available from the corresponding author on reasonable request.

\section{Authors' contributions}

AL and ZZ designed the study. PX, YY, YL and ZL performed the experiments. YX and JC analyzed the bioinformatics data. $\mathrm{PX}$ and $\mathrm{ZZ}$ prepared the manuscript. PX and $\mathrm{ZZ}$ confimed the authenticity of raw data. All authors read and approved the final version of the manuscript.

\section{Ethics approval and consent to participate}

Animal ethical approval was obtained from the Institutional Review Board of The Second Affiliated Hospital of Nanchang University (approval no. A801).

\section{Patient consent for publication}

Not applicable.

\section{Competing interests}

The authors declare that they have no competing interests.

\section{References}

1. Atkins KM, Rawal B, Chaunzwa TL, Lamba N, Bitterman DS, Williams CL, Kozono DE, Baldini EH, Chen AB, Nguyen PL, et al: Cardiac radiation dose, cardiac disease, and mortality in patients with lung cancer. J Am Coll Cardiol 73: 2976-2987, 2019.

2. van Nimwegen FA, Schaapveld M, Cutter DJ, Janus CP, Krol AD, Hauptmann M, Kooijman K, Roesink J, van der Maazen R, Darby SC, et al: Radiation dose-response relationship for risk of coronary heart disease in survivors of hodgkin lymphoma. J Clin Oncol 34: 235-243, 2016.

3. Witt JS, Jagodinsky JC, Liu Y, Yadav P, Kuczmarska-Haas A, Yu M, Maloney JD, Ritter MA, Bassetti MF and Baschnagel AM: Cardiac toxicity in operable esophageal cancer patients treated with or without chemoradiation. Am J Clin Oncol 42: 662-667, 2019

4. Wang $\mathrm{H}, \mathrm{Mu} \mathrm{X}, \mathrm{He} \mathrm{H}$ and Zhang XD: Cancer radiosensitizers. Trends Pharmacol Sci 39: 24-48, 2018.

5. Zamorano JL, Lancellotti P, Rodriguez Muñoz D, Aboyans V, Asteggiano R, Galderisi M, Habib G, Lenihan DJ, Lip GY, Lyon AR, et al; ESC Scientific Document Group: 2016 ESC Position Paper on cancer treatments and cardiovascular toxicity developed under the auspices of the ESC Committee for Practice Guidelines: The Task Force for cancer treatments and cardiovascular toxicity of the European Society of Cardiology (ESC). Eur Heart J 37: 2768-2801, 2016.

6. Schaue D and McBride WH: Opportunities and challenges of radiotherapy for treating cancer. Nat Rev Clin Oncol 12: 527-540, 2015. 
7. Boerma M, Sridharan V, Mao XW, Nelson GA, Cheema AK, Koturbash I, Singh SP, Tackett AJ and Hauer-Jensen M: Effects of ionizing radiation on the heart. Mutat Res Rev Mutat Res 770: 319-327, 2016

8. Bhattacharya $S$ and Asaithamby A: Ionizing radiation and heart risks. Semin Cell Dev Biol 58: 14-25, 2016.

9. Wang H, Wei J, Zheng Q, Meng L, Xin Y, Yin X and Jiang X: Radiation-induced heart disease: A review of classification, mechanism and prevention. Int J Biol Sci 15: 2128-2138, 2019.

10. Raghunathan D, Khilji MI, Hassan SA and Yusuf SW: Radiationinduced cardiovascular disease. Curr Atheroscler Rep 19: 22, 2017.

11. Yusuf SW, Venkatesulu BP, Mahadevan LS and Krishnan S Radiation-induced cardiovascular disease: A clinical perspective. Front Cardiovasc Med 4: 66, 2017

12. Barjaktarovic Z, Shyla A, Azimzadeh O, Schulz S, Haagen J Dörr W, Sarioglu H, Atkinson MJ, Zischka H and Tapio S: Ionising radiation induces persistent alterations in the cardiac mitochondrial function of C57BL/6 mice 40 weeks after local heart exposure. Radiother Oncol 106: 404-410, 2013.

13. Guldner L, Haddy N, Pein F, Diallo I, Shamsaldin A, Dahan M, Lebidois J, Merlet P, Villain E, Sidi D, et al: Radiation dose and long term risk of cardiac pathology following radiotherapy and anthracyclin for a childhood cancer. Radiother Oncol 81: 47-56, 2006.

14. Mezzaroma E, Di X, Graves P, Toldo S, Van Tassell BW, Kannan H, Baumgarten C, Voelkel N, Gewirtz DA and Abbate A: A mouse model of radiation-induced cardiomyopathy. Int J Cardiol 156: 231-233, 2012

15. Dreyfuss AD, Goia D, Shoniyozov K, Shewale SV, Velalopoulou A, Mazzoni S, Avgousti H, Metzler SD, Bravo PE, Feigenberg SJ, et al: A novel mouse model of radiation-induced cardiac injury reveals biological and radiological biomarkers of cardiac dysfunction with potential clinical relevance. Clin Cancer Res 27: 2266-2276, 2021.

16. Zeng ZM, Xu P, Zhou S, Du HY, Jiang XL, Cai J, Huang L and Liu AW: Positive association between heart dosimetry parameters and a novel cardiac biomarker, solubleST-2, in thoracic cancer chest radiation. J Clin Lab Anal 34: e23150, 2020.

17. Adams MJ, Hardenbergh PH, Constine LS and Lipshultz SE: Radiation-associated cardiovascular disease. Crit Rev Oncol Hematol 45: 55-75, 2003

18. Kreuzer M, Auvinen A, Cardis E, Hall J, Jourdain JR, Laurier D, Little MP, Peters A, Raj K, Russell NS, et al: Low-dose ionising radiation and cardiovascular diseases - Strategies for molecular epidemiological studies in Europe. Mutat Res Rev Mutat Res 764 90-100, 2015

19. Sayler E, Dolney D, Avery S and Koch C: Shielding considerations for the small animal radiation research platform (SARRP). Health Phys 104: 471-480, 2013.

20. Hoving S, Seemann I, Visser NL, te Poele JA and Stewart FA: Thalidomide is not able to inhibit radiation-induced heart disease. Int J Radiat Biol 89: 685-691, 2013

21. Wang B, Wang H, Zhang M, Ji R, Wei J, Xin Y and Jiang X Radiation-induced myocardial fibrosis: Mechanisms underlying its pathogenesis and therapeutic strategies. J Cell Mol Med 24: $7717-7729,2020$

22. Slezak J, Kura B, Babal P, Barancik M, Ferko M, Frimmel K, Kalocayova B, Kukreja RC, Lazou A, Mezesova L, et al: Potential markers and metabolic processes involved in the mechanism of radiation-induced heart injury. Can J Physiol Pharmacol 95: 1190-1203, 2017

23. Azzam EI, Jay-Gerin JP and Pain D: Ionizing radiation-induced metabolic oxidative stress and prolonged cell injury. Cancer Lett 327: 48-60, 2012.

24. Zhao W and Robbins ME: Inflammation and chronic oxidative stress in radiation-induced late normal tissue injury: Therapeutic implications. Curr Med Chem 16: 130-143, 2009.

25. Baselet B, Sonveaux P, Baatout S and Aerts A: Pathological effects of ionizing radiation: Endothelial activation and dysfunction. Cell Mol Life Sci 76: 699-728, 2019.

26. Zhang Z, Wu S, Stenoien DL and Paša-Tolić L: High-throughput proteomics. Annu Rev Anal Chem (Palo Alto, Calif) 7: 427-454, 2014.

27. Sharon D, Tilgner H, Grubert F and Snyder M: A single-molecule long-read survey of the human transcriptome. Nat Biotechnol 31 : 1009-1014, 2013

28. Zhang B, Wang J, Wang X, Zhu J, Liu Q, Shi Z, Chambers MC, Zimmerman LJ, Shaddox KF, Kim S, et al; NCI CPTAC: Proteogenomic characterization of human colon and rectal cancer. Nature 513: 382-387, 2014
29. Mun DG, Bhin J, Kim S, Kim H, Jung JH, Jung Y, Jang YE, Park JM, Kim H, Jung Y, et al: Proteogenomic characterization of human early-onset gastric cancer. Cancer Cell 35: 111-124.e10, 2019.

30. Li X, Wang W and Chen J: Recent progress in mass spectrometry proteomics for biomedical research. Sci China Life Sci 60: 1093-1113, 2017.

31. Shen B, Yi X, Sun Y, Bi X, Du J, Zhang C, Quan S, Zhang F, Sun R, Qian L, et al: Proteomic and Metabolomic Characterization of COVID-19 Patient Sera. Cell 182: 59-72.e15, 2020

32. Zeng ZM, Du HY, Xiong L, Zeng XL, Zhang P, Cai J, Huang L and Liu AW: BRCA1 protects cardiac microvascular endothelial cells against irradiation by regulating p21-mediated cell cycle arrest. Life Sci 244: 117342, 2020.

33. Li J, Zhang N, Song LB, Liao WT, Jiang LL, Gong LY, Wu J, Yuan J, Zhang HZ, Zeng MS, et al: Astrocyte elevated gene-1 is a novel prognostic marker for breast cancer progression and overall patient survival. Clin Cancer Res 14: 3319-3326, 2008.

34. Shannon P, Markiel A, Ozier O, Baliga NS, Wang JT, Ramage D, Amin N, Schwikowski B and Ideker T: Cytoscape: A software environment for integrated models of biomolecular interaction networks. Genome Res 13: 2498-2504, 2003.

35. Bandettini WP, Kellman P, Mancini C, Booker OJ, Vasu S, Leung SW, Wilson JR, Shanbhag SM, Chen MY and Arai AE: MultiContrast Delayed Enhancement (MCODE) improves detection of subendocardial myocardial infarction by late gadolinium enhancement cardiovascular magnetic resonance: A clinical validation study. J Cardiovasc Magn Reson 14: 83, 2012.

36. Chin $\mathrm{CH}$, Chen $\mathrm{SH}, \mathrm{Wu} \mathrm{HH}$, Ho CW, Ko MT and Lin CY: cytoHubba: Identifying hub objects and sub-networks from complex interactome. BMC Syst Biol 8 (Suppl 4): S11, 2014.

37. Wang Y, Cai J, Zeng X, Chen Y, Yan W, Ouyang Y, Xiao D, Zeng Z, Huang L and Liu A: Downregulation of toll-like receptor 4 induces suppressive effects on hepatitis B virus-related hepatocellular carcinoma via ERK1/2 signaling. BMC Cancer 15 : 821,2015

38. Azimzadeh O, Sievert W, Sarioglu H, Yentrapalli R Barjaktarovic Z, Sriharshan A, Ueffing M, Janik D, Aichler M, Atkinson MJ, et al: PPAR alpha: A novel radiation target in locally exposed Mus musculus heart revealed by quantitative proteomics. J Proteome Res 12: 2700-2714, 2013.

39. Subramanian V, Seemann I, Merl-Pham J, Hauck SM, Stewart FA, Atkinson MJ, Tapio S and Azimzadeh O: Role of TGF beta and PPAR alpha signaling pathways in radiation response of locally exposed heart: Integrated global transcriptomics and proteomics analysis. J Proteome Res 16: 307-318, 2017.

40. Tsuchida T and Friedman SL: Mechanisms of hepatic stellate cell activation. Nat Rev Gastroenterol Hepatol 14: 397-411, 2017.

41. Zhao X, Kwan JY, Yip K, Liu PP and Liu FF: Targeting metabolic dysregulation for fibrosis therapy. Nat Rev Drug Discov 19: $57-75,2020$

42. Meziani L, Mondini M, Petit B, Boissonnas A, Thomas de Montpreville V, Mercier O, Vozenin MC and Deutsch E: CSF1R inhibition prevents radiation pulmonary fibrosis by depletion of interstitial macrophages. Eur Respir J 51: 51, 2018.

43. Stoddard TJ, Varadarajan VV, Dziegielewski PT, Boyce BJ and Justice JM: Detection of microbiota in post radiation sinusitis. Ann Otol Rhinol Laryngol 128: 1116-1121, 2019.

44. Roy S and Trinchieri G: Microbiota: A key orchestrator of cancer therapy. Nat Rev Cancer 17: 271-285, 2017.

45. Liu LK, Ouyang W, Zhao X, Su ShF, Yang Y, Ding WJ, Luo X, He ZX and Lu B:Pathogenesis and prevention of radiation-induced myocardial fibrosis. Asian Pac J Cancer Prev 18: 583-587, 2017.

46. Soh J, Sugimoto S, Namba K, Miura A, Shiotani T, Yamamoto H, Suzawa K, Shien K, Yamamoto H, Okazaki M, et al: Chronic lung injury after trimodality therapy for locally advanced non-small cell lung cancer. Ann Thorac Surg 112: 279-288, 2020.

47. Annesley SJ and Fisher PR: Mitochondria in health and disease. Cells 8: 8, 2019.

48. Khomich O, Ivanov AV and Bartosch B: Metabolic hallmarks of hepatic stellate cells in liver fibrosis. Cells 9: 9, 2019.

49. Sabbah HN: Targeting the mitochondria in heart failure: A translational perspective. JACC Basic Transl Sci 5: 88-106, 2020.

50. Gollmer J, Zirlik A and Bugger H: Mitochondrial mechanisms in diabetic cardiomyopathy. Diabetes Metab J 44: 33-53, 2020.

51. de Paz-Lugo P, Lupiáñez JA and Meléndez-Hevia E: High glycine concentration increases collagen synthesis by articular chondrocytes in vitro: Acute glycine deficiency could be an important cause of osteoarthritis. Amino Acids 50: 1357-1365, 2018. 
52. Nishikawa T, Bellance N, Damm A, Bing H, Zhu Z, Handa K, Yovchev MI, Sehgal V, Moss TJ, Oertel M, et al: A switch in the source of ATP production and a loss in capacity to perform glycolysis are hallmarks of hepatocyte failure in advance liver disease. J Hepatol 60: 1203-1211, 2014.

53. Zhao YD, Yin L, Archer S, Lu C, Zhao G, Yao Y, Wu L, Hsin M, Waddell TK, Keshavjee S, et al: Metabolic heterogeneity of idiopathic pulmonary fibrosis: A metabolomic study. BMJ Open Respir Res 4: e000183, 2017

54. Im MJ, Freshwater MF and Hoopes JE: Enzyme activities in granulation tissue: Energy for collagen synthesis. J Surg Res 20: $121-125,1976$.

55. Wang SP, Yang H, Wu JW, Gauthier N, Fukao T and Mitchell GA: Metabolism as a tool for understanding human brain evolution: Lipid energy metabolism as an example. J Hum Evol 77: 41-49, 2014

56. Vidali S, Aminzadeh S, Lambert B, Rutherford T, Sperl W, Kofler B and Feichtinger RG: Mitochondria: The ketogenic diet - A metabolism-based therapy. Int J Biochem Cell Biol 63: 55-59, 2015.

57. Brookes PS, Yoon Y, Robotham JL, Anders MW and Sheu SS Calcium, ATP, and ROS: A mitochondrial love-hate triangle. Am J Physiol Cell Physiol 287: C817-C833, 2004.

58. du Plessis SS, Agarwal A, Mohanty G and van der Linde M: Oxidative phosphorylation versus glycolysis: What fuel do spermatozoa use? Asian J Androl 17: 230-235, 2015.

59. Vincent AS, Phan TT, Mukhopadhyay A, Lim HY, Halliwell B and Wong KP: Human skin keloid fibroblasts display bioenergetics of cancer cells. J Invest Dermatol 128: 702-709, 2008.

60. Liu Y, Li Y, Zhang T, Zhao H, Fan S, Cai X, Liu Y, Li Z, Gao S, Li Y, et al: Analysis of biomarkers and metabolic pathways in patients with unstable angina based on ultra high performance liquid chromatography quadrupole time of flight mass spectrometry. Mol Med Rep 22: 3862-3872, 2020.

61. Michel M, Dubowy KO, Zlamy M, Karall D, Adam MG Entenmann A,Keller MA, Koch J, Odri Komazec I, Geiger R, et al: Targeted metabolomic analysis of serum phospholipid and acylcarnitine in the adult Fontan patient with a dominant left ventricle. Ther Adv Chronic Dis: Apr 27, 2020 (Epub ahead of print). doi: $10.1177 / 2040622320916031$
62. Ma CX, Zhao XK and Li YD: New therapeutic insights into radiation-induced myocardial fibrosis. Ther Adv Chronic Dis: Aug 8 , 2019 (Epub ahead of print). doi: 10.1177/2040622319868383

63. Del Re DP, Amgalan D, Linkermann A, Liu Q and Kitsis RN: Fundamental mechanisms of regulated cell death and implications for heart disease. Physiol Rev 99: 1765-1817, 2019.

64. Vásquez-Trincado C, García-Carvajal I, Pennanen C, Parra V, Hill JA, Rothermel BA and Lavandero S: Mitochondrial dynamics, mitophagy and cardiovascular disease. J Physiol 594: 509-525, 2016.

65. Sridharan V, Thomas CJ, Cao M, Melnyk SB, Pavliv O, Joseph J, Singh SP, Sharma S, Moros EG and Boerma M: Effects of local irradiation combined with sunitinib on early remodeling, mitochondria, and oxidative stress in the rat heart. Radiother Oncol 119: 259-264, 2016.

66. Sridharan V, Seawright JW, Antonawich FJ, Garnett M, Cao M, Singh P and Boerma M: Late Administration of a Palladium Lipoic Acid Complex (POLY-MVA) modifies cardiac mitochondria but not functional or structural manifestations of radiation-induced heart disease in a rat model. Radiat Res 187: 361-366, 2017.

67. Dai C, He L, Ma B and Chen T: Facile nanolization strategy for therapeutic ganoderma lucidum spore oil to achieve enhanced protection against radiation-induced heart disease. Small 15: e1902642, 2019.

68. Schlaak RA, Frei A, SenthilKumar G, Tsaih SW, Wells C, Mishra J, Flister MJ, Camara AKS and Bergom C: Differences in expression of mitochondrial complexes due to genetic variants may alter sensitivity to radiation-induced cardiac dysfunction. Front Cardiovasc Med 7: 23, 2020.

69. Ping Z, Peng Y, Lang H, Xinyong C, Zhiyi Z, Xiaocheng W, Hong $Z$ and Liang S: Oxidative stress radiation-induced cardiotoxicity. Oxid Med Cell Longev: Mar 1, 2020 (Epub ahead of print). doi: 10.1155/2020/3579143.

This work is licensed under a Creative Commons Attribution-NonCommercial-NoDerivatives 4.0 International (CC BY-NC-ND 4.0) License. 